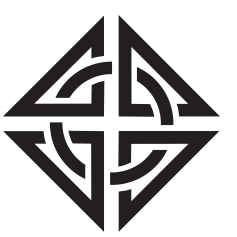

Sharif University of Technology

Scientia Iranica

Transactions A: Civil Engineering

http://scientiairanica.sharif.edu

\title{
Utilizing new spherical Hankel shape functions to reformulate the deflection, free vibration, and buckling analysis of Mindlin plates based on finite element method
}

\author{
M. Mohammadi Nia, S. Shojaee, and S. Hamzehei-Javaran* \\ Department of Civil Engineering, Shahid Bahonar University of Kerman, Kerman, P.O. Box 76169-133, Iran.
}

Received 3 September 2017; received in revised form 20 June 2018; accepted 27 October 2018

\author{
KEYWORDS \\ Spherical Hankel \\ shape functions; \\ Radial basis functions; \\ Mindlin plate theory; \\ Finite element \\ method; \\ Free vibration; \\ Buckling.
}

\begin{abstract}
In this study, a new class of shape functions, namely spherical Hankel shape functions, is derived and applied to reformulate the deflection, free vibration, and buckling of Mindlin plates based on Finite Element Method (FEM). In doing so, the addition of polynomial terms to the functional expansion, in which only spherical Hankel Radial Basis Functions (RBFs) are used, leads to obtaining spherical Hankel shape functions. Accordingly, the application of polynomial and spherical Bessel function fields together results in achieving greater robustness and effectiveness. Spherical Hankel shape functions benefit from some useful properties including infinite piecewise continuity, partition of unity, and Kronecker delta property. In the end, the accuracy of the proposed formulation is investigated through several numerical examples for which the same degrees of freedom are selected in both of the presented formulation and the classical FEM. Finally, it can be concluded that the application of spherical Hankel shape functions ensures achieving higher accuracy than the Lagrangian FEM.
\end{abstract}

(C) 2020 Sharif University of Technology. All rights reserved.

\section{Introduction}

Plate structures are one of the most important structures in engineering and used in many industries like civil engineering, shipbuilding, and aerospace engineering. Such structures including bridge buildings, stadiums, and hydropower stations are among the applications of plates in civil engineering. Therefore, the prevalent use of plates has led engineers to analyze them for design purposes.

\footnotetext{
*. Corresponding author. Fax: +983433257154 E-mail address: s.hamzeheijavaran@uk.ac.ir ( $S$. Hamzehe_Javaran)
}

Until now, few analytical methods have been proposed for solving engineering issues, especially the analysis of plates [1]. These methods provide high accuracy, yet they cannot be implemented in many problems as their geometric and loading conditions are so limited. Besides, analytical solutions may be inaccessible and unfeasible for a large number of problems. The aforementioned limitations have encouraged engineers to use numerical methods and computers for solving various problems. Therefore, researchers have tried to use numerical methods such as Finite Element Method (FEM) [2], Boundary Element Method (BEM) [3], meshless and meshfree methods [46], Petrov-Galerkin method [7], and other methods reported in the literature for analysis purposes. Since the application of computers and numerical methods 
is inevitable nowadays, researchers constantly seek to enhance and boost these methods. To this end, one way may be the use of Radial Basis Functions (RBFs) in numerical methods.

In general, RBFs are applied to estimate multivariable functions by linear combinations of terms depending on a univariate function, which is named RBF [8]. RBFs are categorized into two major classes, namely oscillatory and non-oscillatory. For example, real and complex Fourier RBF [9-11] and J-Bessel RBF [12] are oscillatory kinds, while the Gaussian functions [13], thin-plate splines [14,15], compact supported functions [16-18], multiquadrics [19], and inverse multiquadrics [20] are the non-oscillatory ones.

The Fourier-Bessel series and J-Bessel functions are among the applications of Bessel functions as the basis functions in classical mathematics, and J-Bessel and complex Fourier RBFs were previously suggested by Hamzehei Javaran et al. [9-12]. It is worthwhile to note that the J-Bessel RBF just contains the properties of the first kind of Bessel function. Nonetheless, the first kind of Bessel function usually cannot represent the entire features of a physical phenomenon. Hence, to have both characteristics of the first and second kinds of Bessel function, one way may be the use of Hankel function as it includes the first and second kinds of Bessel function, simultaneously.

The present study attempts to put forward new shape functions that contain the advantages of both RBFs and spherical Bessel functions. Accordingly, an oscillatory RBF based on spherical Hankel functions is applied to derive new spherical Hankel shape functions. The Hankel function is created by combining the first and second kinds of Bessel functions in complex space [21-23]. The application of spherical Hankel shape functions results in profiting from the advantages of complex number space in functional space, which leads to a reduction in both algebraic manipulations and formulations. The proposed shape functions can simply satisfy spherical Bessel function fields and polynomial function fields. Due to their properties, achieving higher accuracy along with lower degrees of freedom is accessible. It should be noted that the application of lower degrees of freedom results in decreasing the computational cost. After that, these shape functions are employed to reformulate the deflection, free vibration, and buckling of the Mindlin plates. In the end, five numerical examples are solved and compared with the analytical solutions as well as classical FEM. It can be seen that the results of the proposed shape functions are in better agreement with analytical solutions than the outcomes of the classic finite element.

\section{Spherical Hankel shape functions}

As a preface to expressing the solution field and geometry approximation, the spherical Hankel shape functions are discussed concisely in this section. The RBFs, which have been introduced up to now based on Bessel functions, only benefit from the first kind of Bessel function (J-Bessel), e.g., J-Bessel RBFs [12]. The solution to Bessel's equation is the most prominent application of both the first and second kinds of Bessel functions, which proves that using them together may ensure greater robustness. The aforementioned discussion brings this matter to mind that whether it is possible to suggest RBFs that benefit from both of the first and second kinds of Bessel functions. Consequently, based on the idea of combining spherical Hankel in the imaginary space, their enrichment for a three-node element in the natural coordinate system is explained, which is used for producing a nine-node element in a two-dimensional space.

\subsection{Enrichment of spherical Hankel RBF}

The enrichment of spherical Hankel RBFs is demonstrated for a three-node element in $\xi$ direction of the natural coordinate. Moreover, this process is expanded for a nine-node element in two dimensions, which is used throughout this paper. In order to enrich a three-node element, polynomial terms are added to the functional expansion that only uses RBFs for the approximation.

$$
\begin{aligned}
w_{h}(x)= & \sum_{i=1}^{n} R_{i}(r) a_{i} \\
& +\sum_{k=1}^{m} P_{k}(x) b_{k}=\overrightarrow{\mathbf{R}}^{T}(r) \overrightarrow{\mathbf{a}}+\overrightarrow{\mathbf{P}}^{T}(x) \overrightarrow{\mathbf{b}}
\end{aligned}
$$

where $n$ and $m$ represent the number of nodes and basis polynomial terms, respectively. Herein, $\overrightarrow{\mathbf{R}}^{T}(r)$, $\overrightarrow{\mathbf{P}}^{T}(x), \overrightarrow{\mathbf{a}}^{T}$, and $\overrightarrow{\mathbf{b}}^{T}$ can be assumed as follows:

$$
\begin{aligned}
& \overrightarrow{\mathbf{R}}^{T}(r)=\left[\begin{array}{llll}
R_{1}(r) & R_{2}(r) & \cdots & R_{n}(r)
\end{array}\right], \\
& \overrightarrow{\mathbf{P}}^{T}(x)=\left[\begin{array}{llll}
P_{1}(x) & P_{2}(x) & \cdots & P_{m}(x)
\end{array}\right], \\
& \overrightarrow{\mathbf{a}}^{T}=\left[\begin{array}{llll}
a_{1} & a_{2} & \cdots & a_{n}
\end{array}\right], \\
& \overrightarrow{\mathbf{b}}^{T}=\left[\begin{array}{llll}
b_{1} & b_{2} & \cdots & b_{m}
\end{array}\right] .
\end{aligned}
$$

Satisfying Eq. (1) on the nodes leads to obtaining the following equation:

$$
\overrightarrow{\hat{w}}=\mathbf{R}_{Q} \overrightarrow{\mathbf{a}}+\mathbf{P}_{m} \overrightarrow{\mathbf{b}}
$$

where:

$$
\mathbf{R}_{Q}=\left[\begin{array}{ccc}
R_{1}\left(r_{1}\right) & \ldots & R_{n}\left(r_{1}\right) \\
\vdots & & \vdots \\
R_{1}\left(r_{n}\right) & \ldots & R_{n}\left(r_{n}\right)
\end{array}\right]_{n \times n},
$$




$$
\mathbf{P}_{m}=\left[\begin{array}{ccc}
P_{1}\left(x_{1}\right) & \ldots & P_{m}\left(x_{1}\right) \\
\vdots & & \vdots \\
P_{1}\left(x_{n}\right) & \ldots & P_{m}\left(x_{n}\right)
\end{array}\right]_{n \times m}
$$

Eq. (3) consists of $n+m$ unknowns, whereas only $n$ equations exist. Accordingly, additional conditions must be applied in order to balance the number of knowns and unknowns. The exclusivity of the solution is assured if the following constraints exist [24]:

$$
\sum_{i=1}^{n} P_{k}\left(x_{i}\right) a_{i}=0, \quad \mathbf{P}_{m}^{T} \overrightarrow{\mathbf{a}}=0 .
$$

Thus, the final set of equations is given in the following form:

$$
\left[\begin{array}{cc}
\mathbf{R}_{Q} & \mathbf{P}_{m} \\
\mathbf{P}_{m}^{T} & 0
\end{array}\right]\left[\begin{array}{l}
\overrightarrow{\mathbf{a}} \\
\overrightarrow{\mathbf{b}}
\end{array}\right]=\left[\begin{array}{c}
\overrightarrow{\hat{w}} \\
0
\end{array}\right]
$$

Following some algebraic manipulations, the results below are obtained for the unknown vectors $\overrightarrow{\mathbf{a}}$ and $\overrightarrow{\mathbf{b}}$

$$
\overrightarrow{\mathbf{a}}=\mathbf{S}_{a} \overrightarrow{\hat{w}}, \quad \overrightarrow{\mathbf{b}}=\mathbf{S}_{b} \overrightarrow{\hat{w}}
$$

in which,

$$
\begin{aligned}
& \mathbf{S}_{a}=\mathbf{R}_{Q}^{-1}-\mathbf{R}_{Q}^{-1} \mathbf{P}_{m} \mathbf{S}_{b}, \\
& \mathbf{S}_{b}=\left[\mathbf{P}_{m}^{T} \mathbf{R}_{Q}^{-1} \mathbf{P}_{m}\right]^{-1} \mathbf{P}_{m}^{T} \mathbf{R}_{Q}^{-1} .
\end{aligned}
$$

By replacing $\mathbf{c}$ and $\mathbf{d}$ into Eq. (1), $\overrightarrow{w_{h}}(x)$ becomes:

$$
\overrightarrow{w_{h}}(x)=\left[\mathbf{R}^{T}(r) \mathbf{S}_{a}+\mathbf{P}^{T}(x) \mathbf{S}_{b}\right] \overrightarrow{\hat{w}} .
$$

In Eq. (9), the expression of $\left[\mathbf{R}^{T}(r) \mathbf{S}_{a}+\mathbf{P}^{T}(x) \mathbf{S}_{b}\right]$ relates the functional field to the nodal values. In other words, $\left[\mathbf{R}^{T}(r) \mathbf{S}_{a}+\mathbf{P}^{T}(x) \mathbf{S}_{b}\right]$ is defining shape functions. Hence, the matrix of shape functions is offered as follows:

$$
\boldsymbol{\Phi}(x)=\mathbf{R}^{T}(r) \mathbf{S}_{a}+\mathbf{P}^{T}(x) \mathbf{S}_{b} .
$$

As mentioned in the previous discussions, the demanded RBF can be accessible as in the following form:

$$
R(r)=(e r)^{\tilde{n}+1} h_{\widetilde{n}}^{(1)}(e r) \quad e>0, \quad \tilde{n}=0,1,2, \ldots,
$$

where $n$ and $e$ are the shape parameters of Hankel RBF, and $h_{\widetilde{n}}^{(1)}(e r)=\sqrt{\frac{\pi}{2(e r)}} H_{\widetilde{n}+\frac{1}{2}}^{(1)}(e r)$ indicates the spherical Hankel function of the first kind.

The first order of spherical Hankel function, $h_{\widetilde{n}}^{(1)}(e r)=j_{\widetilde{n}}(e r)+i y_{\widetilde{n}}(e r)$, has singularity at its imaginary part, which is the second kind of spherical Bessel function of order $n$. Therefore, $(e r)^{\tilde{n}+1}$ is employed to eliminate this singularity. Thus, the limiting values of the RBF may be calculated as follows:

$$
\begin{aligned}
& \varphi=\lim _{r \rightarrow 0}(e r)^{\widetilde{n}+1}, \\
& h_{\widetilde{n}}^{(1)}(e r)=0+\frac{(-1)^{\widetilde{n}+1} \times 2^{\widetilde{n}} \times \sqrt{\pi}}{\Gamma\left(-\widetilde{n}+\frac{1}{2}\right)} i,
\end{aligned}
$$

where $\Gamma$ denotes the well-known gamma function. After applying some algebraic manipulations for a threenode element in $\xi$ direction of the natural coordinate system, the desired vectors and matrices are calculated as in the following forms:

$$
\begin{aligned}
& \mathbf{R}(r)=\left[\begin{array}{l}
R_{1}(r) \\
R_{2}(r) \\
R_{3}(r)
\end{array}\right] \\
& =\left[\begin{array}{cc}
\left(e\left|\xi-\xi_{1}\right|\right)^{\tilde{n}+1} h_{n}^{(1)} & \left(e\left|\xi-\xi_{1}\right|\right) \\
\left(e\left|\xi-\xi_{2}\right|\right)^{\tilde{n}+1} h_{n}^{(1)} & \left(e\left|\xi-\xi_{2}\right|\right) \\
\left(e\left|\xi-\xi_{3}\right|\right)^{\tilde{n}+1} h_{n}^{(1)} & \left(e\left|\xi-\xi_{3}\right|\right)
\end{array}\right], \\
& \overrightarrow{\mathbf{P}}(\xi)=\left[\begin{array}{l}
1 \\
\xi
\end{array}\right] \\
& \mathbf{R}_{Q}=\left[\begin{array}{lll}
R_{1}\left(r_{1}\right) & R_{2}\left(r_{1}\right) & R_{3}\left(r_{1}\right) \\
R_{1}\left(r_{2}\right) & R_{2}\left(r_{2}\right) & R_{3}\left(r_{2}\right) \\
R_{1}\left(r_{3}\right) & R_{2}\left(r_{3}\right) & R_{3}\left(r_{3}\right)
\end{array}\right] \\
& =\left[\begin{array}{cc}
\varphi & \left(e\left|\xi_{1}-\xi_{2}\right|\right)^{\tilde{n}+1} h_{\widetilde{n}}^{(1)}\left(e\left|\xi_{1}-\xi_{2}\right|\right) \\
\text { Sym. } & \varphi
\end{array}\right. \\
& \left.\begin{array}{c}
\left(e\left|\xi_{1}-\xi_{3}\right|\right)^{\tilde{n}+1} h_{\widetilde{n}}^{(1)}\left(e\left|\xi_{1}-\xi_{3}\right|\right) \\
\left(e\left|\xi_{2}-\xi_{3}\right|\right)^{\tilde{n}+1} h_{\widetilde{n}}^{(1)}\left(e\left|\xi_{2}-\xi_{3}\right|\right) \\
\varphi
\end{array}\right] \\
& \mathbf{P}_{m}=\left[\begin{array}{ll}
P_{1}\left(\xi_{1}\right) & P_{2}\left(\xi_{1}\right) \\
P_{1}\left(\xi_{2}\right) & P_{2}\left(\xi_{2}\right) \\
P_{1}\left(\xi_{3}\right) & P_{2}\left(\xi_{3}\right)
\end{array}\right]=\left[\begin{array}{ll}
1 & \xi_{1} \\
1 & \xi_{2} \\
1 & \xi_{3}
\end{array}\right] \\
& \mathbf{S}_{c}=\frac{c}{2}\left[\begin{array}{ccc}
1 & -2 & 1 \\
& 4 & -2 \\
\text { sym. } & & 1
\end{array}\right] \text {, } \\
& \mathbf{S}_{d}=\frac{1}{2}\left[\begin{array}{ccc}
\bar{c} & 2(1-\bar{c}) & \bar{c} \\
-1 & 0 & 1
\end{array}\right] \text {. }
\end{aligned}
$$

It should be noted that in Eq. (15), it is one of the properties of RBFs to make the coefficient matrix symmetric. In other words, $R_{i}\left(r_{j}\right)=R_{j}\left(r_{i}\right)$. In Eqs. (17) and (18), $c$ and $\bar{c}$ are complex constants, which are defined below:

$$
c=\frac{1}{(2 e)^{\tilde{n}+1} h_{\widetilde{n}}^{(1)}(2 e)-4(e)^{\tilde{n}+1} h_{\widetilde{n}}^{(1)}(e)+3 \varphi},
$$




$$
\bar{c}=2 c\left[(-e)^{\widetilde{n}+1} h_{\widetilde{n}}^{(1)}(e)+\varphi\right]
$$

where $\varphi=\lim _{r \rightarrow 0} R(r)$, which is already bound by eliminating singularity (Eq. (12)), leads to having no singularity in $R(r)$. To sum up, the shape functions for a three-node element in $\xi$ direction of the natural coordinate system are stated as follows:

$$
\begin{aligned}
& \Phi(\xi)\left[\begin{array}{ll}
\Phi_{1}(\xi) & \Phi_{2}(\xi) \quad \Phi_{3}(\xi)
\end{array}\right] \\
& \Phi_{1}(\xi)=\frac{1}{2}(-\xi+\bar{c}+h(\xi)) \\
& \Phi_{2}(\xi)=(1-\bar{c})-h(\xi) \\
& \Phi_{3}(\xi)=\frac{1}{2}(\xi+\bar{c}+h(\xi))
\end{aligned}
$$

in which:

$$
\begin{aligned}
h(\xi)= & c(e)^{\widetilde{n}+1}\left[(|\xi+1|)^{\widetilde{n}+1} h_{\widetilde{n}}^{(1)}(e|\xi+1|)-2(|\xi|)^{\widetilde{n}+1}\right. \\
& \left.h_{\widetilde{n}}^{(1)}(e|\xi|)+(|\xi-1|)^{\widetilde{n}+1} h_{\widetilde{n}}^{(1)}(e|\xi-1|)\right] . \quad(25)
\end{aligned}
$$

A one-dimensional element with three nodes is shown in Figure 1. Moreover, Figures 2, 3, and 4 demonstrate the real part of spherical Hankel shape functions for a three-node element with various shape parameters $(e$ and $\tilde{n}$ ). It should be noted that the imaginary part is ineffective in the calculations. This issue is described to a greater extent in the following sections.

To expand the shape functions for twodimensional elements, one-dimensional shape functions in the other direction of natural coordinates $\eta$ are defined the same as $\xi$ direction:

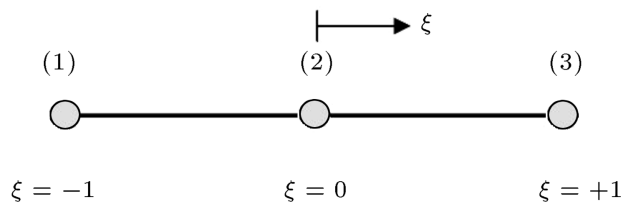

Figure 1. One-dimensional element with three nodes.

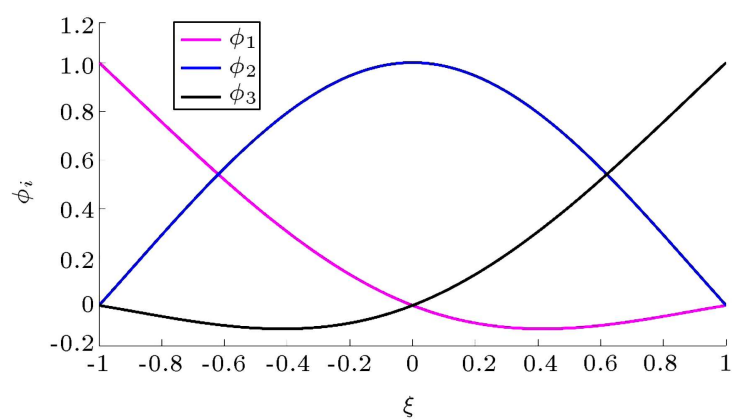

Figure 2. Spherical Hankel shape functions for a three-node element when $\widetilde{n}=1$ and $e=1$.

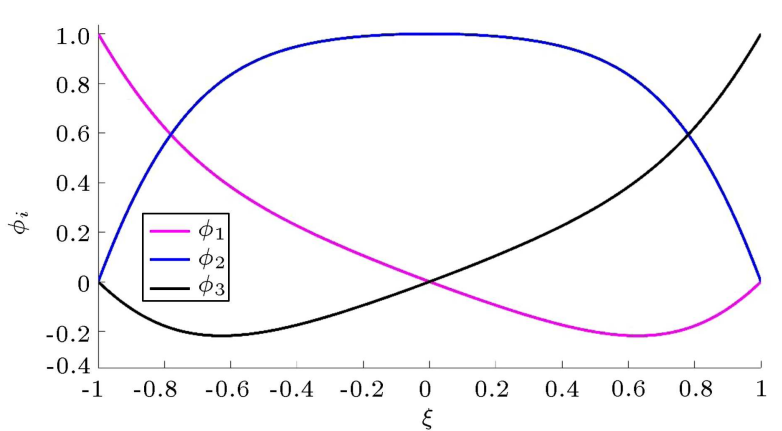

Figure 3. Spherical Hankel shape functions for a three-node element when $\tilde{n}=7$ and $e=4$.

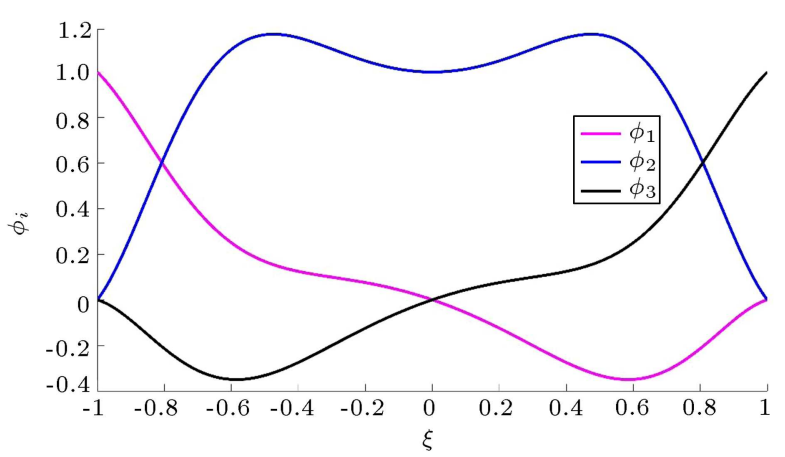

Figure 4. Spherical Hankel shape functions for a three-node element when $\tilde{n}=5$ and $e=6$.

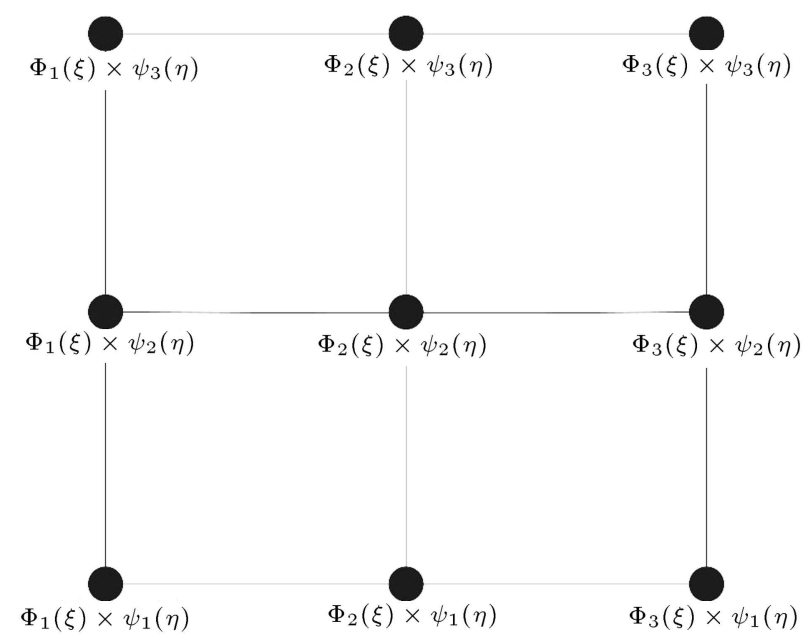

Figure 5. A two-dimensional element with nine nodes.

$$
\psi(\eta)=\left[\begin{array}{lll}
\psi_{1}(\eta) & \psi_{2}(\eta) & \psi_{3}(\eta)
\end{array}\right]
$$

By multiplying shape functions in $\xi$ and $\eta$ directions, shape functions for an element in two dimensions with nine nodes could be produced. Figure 5 shows how to construct a 2D nine-node element.

\subsection{Derivatives of spherical Hankel shape functions}

Derivatives of spherical Hankel shape functions for a three-node element can be obtained as follows: 


$$
\begin{array}{ll}
\Phi_{1}(\xi)=\frac{1}{2}(-\xi+\bar{c}+h(\xi)), & \Phi_{2}(\xi)=(1-\bar{c})-h(\xi), \\
\Phi_{3}(\xi)=\frac{1}{2}(\xi+\bar{c}+h(\xi)), & \Phi_{1}^{\prime}(\xi)=\frac{1}{2}\left(-1+h^{\prime}(\xi)\right), \\
\Phi_{2}^{\prime}(\xi)=-h^{\prime}(\xi) & \Phi_{3}^{\prime}(\xi)=\frac{1}{2}\left(1+h^{\prime}(\xi)\right) \\
\Phi_{1}^{\prime \prime}(\xi)=\frac{h^{\prime \prime}(\xi)}{{h^{\prime \prime}}^{2}(\xi)} & \Phi_{2}^{\prime \prime}(\xi)=-h^{\prime \prime}(\xi), \\
\Phi_{3}^{\prime \prime}(\xi)=\frac{h^{\prime \prime \prime}(\xi)}{2} & \Phi_{1}^{\prime \prime \prime}(\xi)=\frac{h^{\prime \prime \prime}}{2}(\xi) \\
\Phi_{2}^{\prime \prime \prime}(\xi)=-h^{\prime \prime \prime}(\xi) & \Phi_{3}^{\prime \prime \prime}(\xi)=\frac{h^{\prime \prime}}{2} \cdots
\end{array}
$$

According to the properties of spherical Hankel functions, there is a recurrence relation between their derivatives, which can be expressed as follows:

$$
\left(\frac{d}{x d x}\right)^{p}\left[x^{\widetilde{n}+1} h_{\widetilde{n}}^{(1)}(x)\right]=x^{\widetilde{n}-p+1} h_{\widetilde{n}-p}^{(1)}(x) .
$$

For example, by substituting Eq. (28) into Eq. (25), the first three derivatives of $h(\xi)$ can be obtained by the following equation:

$$
\begin{aligned}
& h(\xi)=c e^{\widetilde{n}+1}\left[(1+\xi)^{\tilde{n}+1} h_{\widetilde{n}}^{(1)}(e(1+\xi))\right. \\
& -2|\xi|^{\widetilde{n}+1} h_{\widetilde{n}}^{(1)}(e|\xi|)+(1-\xi)^{\widetilde{n}+1} h_{\widetilde{n}}^{(1)} \\
& (e(1-\xi))] \\
& h^{\prime}(\xi)=c e^{\widetilde{n}+2}\left[(1+\xi)^{\widetilde{n}+1} h_{\widetilde{n}-1}^{(1)}(e(1+\xi))\right. \\
& -2(H(\xi)-H(-\xi))|\xi|^{\tilde{n}+1} h_{\tilde{n}-1}^{(1)}(e|\xi|) \\
& \left.-(1-\xi)^{\widetilde{n}+1} h_{\widetilde{n}-1}^{(1)}(e(1-\xi))\right], \\
& h^{\prime \prime}(\xi)=c\left(e ^ { \tilde { n } + 3 } \left[(1+\xi)^{\tilde{n}+1} h_{\tilde{n}-2}^{(1)}(e(1+\xi))\right.\right. \\
& -2(H(\xi)+H(-\xi))|-\xi|^{\tilde{n}+1} h_{\tilde{n}-2}^{(1)}(e|\xi|) \\
& \left.+(1-\xi)^{\tilde{n}+1} h_{\tilde{n}-2}^{(1)}(e(1-\xi))\right] \\
& +e^{\tilde{n}+2}\left[(1+\xi)^{\tilde{n}} h_{\tilde{n}-1}^{(1)}(e(1+\xi))\right. \\
& -2(H(\xi)+H(-\xi))|\xi|^{\tilde{n}} h_{\tilde{n}-1}^{(1)}(e|\xi|) \\
& \left.\left.+(1-\xi)^{\tilde{n}} h_{\tilde{n}-1}^{(1)}(e(1-\xi))\right]\right) \text {, } \\
& h^{\prime \prime \prime}(\xi)=c\left(e ^ { \widetilde { n } + 4 } \left[(1+\xi)^{\widetilde{n}+1} h_{\widetilde{n}-3}^{(1)}(e(1+\xi))\right.\right. \\
& -2(H(\xi)-H(-\xi))|\xi|^{\tilde{n}+1} h_{\tilde{n}-3}^{(1)}(e|\xi|) \\
& \left.-(1-\xi)^{\widetilde{n}+1} h_{\widetilde{n}-3}^{(1)}(e(1-\xi))\right]
\end{aligned}
$$

$$
\begin{aligned}
& +3 \varepsilon^{\widetilde{n}+3}\left[(1+\xi)^{\widetilde{n}} h_{\widetilde{n}-2}^{(1)}(e(1+\xi))\right. \\
& -2(H(\xi)-H(-\xi))|\xi|^{\widetilde{n}} h_{\widetilde{n}-2}^{(1)}(e|\xi|) \\
& \left.\left.-(1-\xi)^{\widetilde{n}} h_{\widetilde{n}-2}^{(1)}(e(1-\xi))\right]\right),
\end{aligned}
$$

in which $H$ represents the well-known Heaviside step function.

\subsection{Properties of spherical Hankel shape functions}

Some properties of spherical Hankel shape functions can be summarized as follows:

i. Kronecker delta property, $\Phi_{m}\left(\xi_{n}\right)=\delta_{m n}(1+0 i)$, where $i=\sqrt{-1}$ and $\delta_{m n}$ is the Kronecker delta symbol;

ii. Partition of unity, $\sum_{1}^{3} \Phi_{j}(\xi)=1+0 i$;

iii. Infinite piecewise continuity: being in the form of Bessel functions, they can be derived infinitely;

iv. The linear independence property, which is easily derived from Kronecker delta property according to applied mathematics.

\section{Formulation of Mindlin plates based on spherical Hankel shape functions}

This theory considers shear deformations; thus, deflection and rotations are independent. Thus, the stiffness matrix consists of bending and shear terms. The Mindlin element equation is given as follows (for more details, see [25]):

$$
\left(\mathbf{K}_{b}+\mathbf{K}_{s}\right) \overrightarrow{\mathbf{d}}=\overrightarrow{\mathbf{r}}
$$

where $\overrightarrow{\mathbf{d}}$ and $\overrightarrow{\mathbf{r}}$ indicate the displacement and equivalent nodal load vectors. Moreover, $\overrightarrow{\mathbf{r}}$ can be obtained as follows:

$$
\overrightarrow{\mathbf{r}}=\int_{\Omega} \overrightarrow{\mathbf{f}} \mathbf{N} d \Omega
$$

in which $\mathbf{N}$ and $\overrightarrow{\mathbf{f}}$ are the shape function matrix and the load vector, respectively. It should be noted that $\Omega$ is liable to change according to the nature of the load (for more details, see [25]). Besides, bending and shear stiffness matrices can be calculated through the following equations:

$$
\begin{aligned}
\mathbf{K}_{b} & =\iint_{A} \mathbf{B}_{b} \mathbf{C B}_{b}^{T} d A, \\
\mathbf{K}_{s} & =k \mathbf{G} h \iint_{A} \mathbf{B}_{s} \mathbf{B}_{s}^{T} d A,
\end{aligned}
$$




$$
\mathbf{C}=\frac{E}{1-\nu^{2}}\left[\begin{array}{ccc}
1 & \nu & 0 \\
\nu & 1 & 0 \\
0 & 0 & \frac{1-\nu}{2}
\end{array}\right],
$$

where $k$ is shear correction factor, $\mathbf{G}$ shear modulus, $h$ the thickness of the plate, $\nu$ Poisson's ratio, and $E$ Young's modulus.

Now, the main challenge is the selection of shape functions. Is it possible to use the spherical Hankel shape functions in Eqs. (30)-(32)? To do so, the matrices $\mathbf{N}, \mathbf{B}_{b}$, and $\mathbf{B}_{s}$ from Eqs. (31)-(33) for a ninenode element using Hankel shape functions are given in the following forms:

$$
\mathbf{N}=\left[\begin{array}{ccccccc}
R_{1} & R_{2} & \cdots & R_{9} & 0 & 0 & \\
0 & 0 & \cdots & 0 & R_{1} & R_{2} & \\
0 & 0 & \cdots & 0 & 0 & 0 & \\
& \cdots & 0 & 0 & 0 & \cdots & 0 \\
& \cdots & R_{9} & 0 & 0 & \cdots & 0 \\
& \cdots & 0 & R_{1} & R_{2} & \cdots & R_{9}
\end{array}\right]_{3 \times 27} .
$$

$\mathbf{B}_{b}=\left[\begin{array}{ccccccc}0 & \frac{\partial R_{1}}{\partial x} & 0 & \cdots & 0 & \frac{\partial R_{9}}{\partial x} & 0 \\ 0 & 0 & \frac{\partial R_{1}}{\partial y} & \cdots & 0 & 0 & \frac{\partial R_{9}}{\partial y} \\ 0 & \frac{\partial R_{1}}{\partial y} & \frac{\partial R_{1}}{\partial x} & \cdots & 0 & \frac{\partial R_{9}}{\partial y} & \frac{\partial R_{9}}{\partial x}\end{array}\right]_{3 \times 27}$,

$$
\mathbf{B}_{s}=\left[\begin{array}{ccccccc}
\frac{\partial R_{1}}{\partial x} & R_{1} & 0 & \cdots & \frac{\partial R_{9}}{\partial x} & R_{9} & 0 \\
\frac{\partial R_{1}}{\partial y} & 0 & R_{1} & \cdots & \frac{\partial R_{9}}{\partial y} & 0 & R_{9}
\end{array}\right]_{2 \times 27} \underset{(37)}{ }
$$

where $R$ denotes Hankel shape functions. For example, $R_{1}=\Phi_{1}(\xi) \times \psi_{1}(\eta), R_{2}=\Phi_{2}(\xi) \times \psi_{1}(\eta), R_{3}=$ $\Phi_{3}(\xi) \times \psi_{1}(\eta), R_{4}=\Phi_{1}(\xi) \times \psi_{2}(\eta)$, and so on (for more details, see Figure 5$)$.

\section{Free vibration formulation of Mindlin plates based on spherical Hankel shape functions}

Based on Hamilton's principle, the motion equation of Mindlin plate is obtained below (for more details, see $[26])$ :

$$
\mathbf{M} \ddot{\mathbf{U}}+\mathbf{K U}=0,
$$

where $\mathbf{U}$ and $\ddot{\mathbf{U}}$ are displacements and accelerations, and $\mathbf{M}$ and $\mathbf{K}$ represent the mass and stiffness matrices (Eqs. (32)-(37)), respectively. The mass matrix is expressed as follows:

$$
\mathbf{M}=\int_{A} \rho \mathbf{N}^{T}\left[\begin{array}{ccc}
h & 0 & 0 \\
0 & \frac{h^{3}}{12} & 0 \\
0 & 0 & \frac{h^{3}}{12}
\end{array}\right] \mathbf{N} d A
$$

where $\rho$ is the mass density. By implementing the eigenvalue problem, the following equation is obtained:

$$
\left(\mathbf{K}-\omega^{2} \mathbf{M}\right) \overrightarrow{\mathbf{X}}=0
$$

in which $\omega$ is natural frequency, and $\overrightarrow{\mathbf{X}}$ is the mode shape vector.

\section{Buckling formulation of Mindlin plates based on spherical Hankel shape functions}

In this section, the buckling of Mindlin plates is formulated based on spherical Hankel as the shape functions. The stability problem contains the solution of the following equation (for more details, see [26]):

$$
\left[\mathbf{K}-\lambda \mathbf{K}_{G}\right] \mathbf{a}^{i}=0, \quad i=1,2, \ldots, r,
$$

where $\lambda$ denotes a constant that increases in-plane loads in order that the buckling happens, and $\mathbf{a}^{i}$ represents buckling mode shapes. In addition, $\mathbf{K}_{G}$ implies the geometric stiffness matrix, for which spherical Hankel is used for interpolation as follows:

$$
\mathbf{K}_{G}=\mathbf{K}_{G b}+\mathbf{K}_{G s},
$$

where $\mathbf{K}_{G b}$ and $\mathbf{K}_{G s}$ are the bending and shear geometric matrices, respectively, which can be expressed as follows:

$$
\begin{aligned}
\mathbf{K}_{G b}= & \int_{-1}^{+1} \int_{-1}^{+1} \mathbf{G}_{b}{ }^{T} \hat{\sigma}_{0}^{T} \mathbf{G}_{b} h \operatorname{det}(\mathbf{J}) d \xi d \eta, \\
\mathbf{K}_{G s}= & \int_{-1}^{+1} \int_{-1}^{+1} \mathbf{G}_{s 1}{ }^{T} \hat{\sigma}_{0} \mathbf{G}_{s 1} \operatorname{det}(\mathbf{J}) d \xi d \eta \\
& +\int_{-1}^{+1} \int_{-1}^{+1} \mathbf{G}_{s 2}{ }^{T} \hat{\sigma}_{0} \mathbf{G}_{s 2} \frac{h^{3}}{12} \operatorname{det}(\mathbf{J}) d \xi d \eta,
\end{aligned}
$$

in which $\mathbf{J}$ is the well-known Jacobian matrix, and $\hat{\sigma}_{0}^{T}$ stands for the initial stress field, which can be considered as follows:

$$
\hat{\sigma}_{0}^{T}=\left[\begin{array}{cc}
\sigma_{x}^{0} & \tau_{x y}^{0} \\
\tau_{x y}^{0} & \sigma_{y}^{0}
\end{array}\right] .
$$

Furthermore, for the particular node $i, \mathbf{G}_{b}, \mathbf{G}_{s 1}$, and $\mathbf{G}_{s 2}$ can be expressed as follows:

$$
\begin{aligned}
\mathbf{G}_{b} & =\left[\begin{array}{ccc}
\frac{\partial R_{i}}{\partial x} & 0 & 0 \\
\frac{\partial R_{i}}{\partial y} & 0 & 0
\end{array}\right], \\
\mathbf{G}_{s 1} & =\left[\begin{array}{lll}
0 & \frac{\partial R_{i}}{\partial x} & 0 \\
0 & \frac{\partial R_{i}}{\partial y} & 0
\end{array}\right], \\
\mathbf{G}_{s 2} & =\left[\begin{array}{lll}
0 & 0 & \frac{\partial R_{i}}{\partial x} \\
0 & 0 & \frac{\partial R_{i}}{\partial y}
\end{array}\right] .
\end{aligned}
$$




\section{Numerical examples}

In this part, to demonstrate the validity and robustness of the aforesaid formulations, five various numerical examples are provided. The offered formulation results are compared with available results in the literature and classical finite element using Q9 elements. Throughout numerical examples, errors (percentage relative errors) are calculated as follows:

$$
\text { Error }=\left|\frac{\omega^{\text {num }}-\omega^{\text {exact }}}{\omega^{\text {exact }}}\right| \times 100 \text {. }
$$

Besides, the following parameters are considered: Young's modulus $E=10920$, Poisson's ratio $\nu=0.3$, and material density $\rho=1$.

\subsection{Example 1: A square Mindlin plate in bending}

In the first numerical example, a square plate isconsidered with thickness $t$ and side $a$ under uniform distributed load $(P=1)$ with simple and clamped boundary conditions. The non-dimensional deformation is taken as $\bar{w}=w \frac{D}{P a^{4}}$ in which $D=\frac{E t^{3}}{12\left(1-\nu^{2}\right)}$ and $w$ is deflection [26].

In this example, it should be noted that the computational cost is reduced by selecting coarse meshes. Besides, the results of the proposed formulation are compared with the classical finite element as well as analytical ones. Through this example, it is understood that, with low degrees of freedom in the proposed formulation, very better accuracy is reachable than the classical finite element. For instance, in Table 1, when $a / t=10$ and $1 \times 1$ meshes are selected, the error in

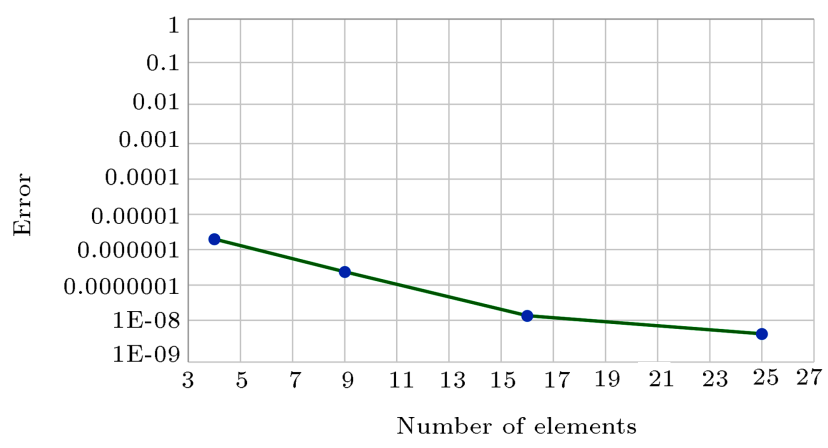

Figure 6. Convergence trend for the fully clamped case.

the presented formulation is $6.7905 e-0.6 \%$, while it is $2.2457 \%$ in the classical FEM.

Tables 1 and 2 show the non-dimensional deformation for SSSS and CCCC boundary conditions, respectively, and different length-to-thickness ratios.

In this example, the fully clamped case is solved with various meshes to show the convergence trend for Hankel shape functions (Figure 6). As the figure shows, considering more elements leads to better accuracy.

\subsection{Example 2: Free vibration of square plate} Free vibration of a square plate with side $a$ and thickness $t$ is considered as the second numerical example. In this case, various boundary conditions and lengthto-thickness ratios are investigated. The dimensionless frequency is considered $\bar{\omega}=\omega a \sqrt{\frac{\rho}{\mathbf{G}}}$, where $\mathbf{G}$ is the shear modulus [27].

In the case of $\mathrm{CCCC}$, the shear correction factor is considered as $\kappa=0.8601$, whereas in the cases of SCSC and SSSS, this factor is taken as $\kappa=0.822$ and $\kappa=5 / 6$, respectively (for more details, see [26]). This

Table 1. Dimensionless deformation of square plate under uniform distributed load $(P=1)$ with simple boundary conditions (SSSS).

\begin{tabular}{ccccccc}
\hline $\boldsymbol{a} / \boldsymbol{t}$ & Mesh & $\begin{array}{c}\text { Present } \\
\text { formulation }\end{array}$ & Error (\%) & Classical FEM & Error (\%) & Analytical [26] \\
\hline \multirow{2}{*}{10} & $1 * 1$ & 0.0042 & $6.7905 \mathrm{E}-06$ & 0.0041 & 2.2457 & 0.00427 \\
& $2 * 2$ & 0.0042 & $4.7143 \mathrm{E}-06$ & 0.0044 & 3.8181 & \\
& & & & & & \\
10000 & $1 * 1$ & 0.0040 & $1.1502 \mathrm{E}-05$ & 0.0039 & 3.7869 & 0.00406 \\
\hline
\end{tabular}

Table 2. Dimensionless deformation of square plate under uniform distributed load $(P=1)$ with clamped boundary conditions (CCCC).

\begin{tabular}{ccccccc}
\hline $\boldsymbol{a} / \boldsymbol{t}$ & Mesh & $\begin{array}{c}\text { Present } \\
\text { formulation }\end{array}$ & Error (\%) & Classical FEM & Error (\%) & Analytical [26] \\
\hline \multirow{2}{*}{10000} & $2 * 2$ & 0.00126 & 0.0002 & 0.00156 & 24.0079 & 0.00126 \\
& $3 * 3$ & 0.00126 & $2.35 \mathrm{E}-05$ & 0.00128 & 2.2514 & \\
\hline
\end{tabular}


example was also investigated by Liew et al. [27] using energy method. To compare the results of the present formulation with analytical ones and classic FEM, the results are shown in tables, and errors are calculated based on these analytical solutions. A $4 \times 4$ mesh is employed for both of the presented formulation and classical FEM.

Tables 3 and 4 show the first eight natural frequency parameters for Mindlin plate with completely simple and clamped boundary conditions, respectively.

Table 3. Dimensionless natural frequencies $\bar{\omega}$ of simply supported square plate.

\begin{tabular}{|c|c|c|c|c|c|c|}
\hline$t / a$ & Mode no & $\begin{array}{c}\text { Present } \\
\text { formulation }\end{array}$ & Error (\%) & Classical FEM & Error (\%) & Analytical [28] \\
\hline \multirow{8}{*}{0.1} & 1 & 0.9228 & 0.7678 & 0.9311 & 0.1283 & 0.930 \\
\hline & 2 & 2.1880 & 1.3953 & 2.2444 & 1.1487 & 2.219 \\
\hline & 3 & 2.1880 & 1.3953 & 2.2444 & 1.1487 & 2.219 \\
\hline & 4 & 3.3535 & 1.5388 & 3.4513 & 1.3328 & 3.406 \\
\hline & 5 & 4.1716 & 0.5450 & 4.3781 & 5.5230 & 4.149 \\
\hline & 6 & 4.1716 & 0.5450 & 4.3781 & 5.5230 & 4.149 \\
\hline & 7 & 5.2051 & 0.0155 & 5.4404 & 4.5038 & 5.206 \\
\hline & 8 & 5.2051 & 0.0155 & 5.4404 & 4.5038 & 5.206 \\
\hline \multirow{8}{*}{0.01} & 1 & 0.0954 & 0.8494 & 0.0963 & 0.0837 & 0.0963 \\
\hline & 2 & 0.2368 & 1.5642 & 0.2435 & 1.2130 & 0.2406 \\
\hline & 3 & 0.2368 & 1.5642 & 0.2435 & 1.2130 & 0.2406 \\
\hline & 4 & 0.3781 & 1.7119 & 0.3904 & 1.5058 & 0.3847 \\
\hline & 5 & 0.4833 & 0.5486 & 0.5111 & 6.3271 & 0.4807 \\
\hline & 6 & 0.4833 & 0.5486 & 0.5111 & 6.3271 & 0.4807 \\
\hline & 7 & 0.6244 & 0.0232 & 0.6577 & 5.3142 & 0.6246 \\
\hline & 8 & 0.6244 & 0.0232 & 0.6577 & 5.3142 & 0.6246 \\
\hline
\end{tabular}

Table 4. Dimensionless natural frequencies $\bar{\omega}$ of fully clamped square plate.

\begin{tabular}{|c|c|c|c|c|c|c|}
\hline$t / a$ & Mode no & $\begin{array}{c}\text { Present } \\
\text { formulation }\end{array}$ & Error (\%) & Classical FEM & Error (\%) & Liew et al. $[27]$ \\
\hline \multirow{8}{*}{0.1} & 1 & 1.5562 & 0.1231 & 1.5959 & 2.4258 & 1.5582 \\
\hline & 2 & 2.9644 & 1.7799 & 3.1018 & 2.7725 & 3.0182 \\
\hline & 3 & 2.9644 & 1.7799 & 3.1018 & 2.7725 & 3.0182 \\
\hline & 4 & 4.1558 & 0.3646 & 4.3512 & 4.3201 & 4.1711 \\
\hline & 5 & 5.0728 & 0.9554 & 5.4152 & 5.7288 & 5.1218 \\
\hline & 6 & 5.1237 & 0.6908 & 5.4706 & 6.0323 & 5.1594 \\
\hline & 7 & 6.0826 & 1.0780 & 6.4453 & 7.1044 & 6.0178 \\
\hline & 8 & 6.0826 & 1.0780 & 6.4453 & 7.1044 & 6.0178 \\
\hline \multirow{8}{*}{0.01} & 1 & 0.1707 & 2.0248 & 0.1760 & 0.9860 & 0.1743 \\
\hline & 2 & 0.3464 & 3.1246 & 0.3668 & 2.5949 & 0.3576 \\
\hline & 3 & 0.3464 & 3.1246 & 0.3668 & 2.5949 & 0.3576 \\
\hline & 4 & 0.5097 & 2.7195 & 0.5411 & 3.2683 & 0.524 \\
\hline & 5 & 0.6496 & 0.4882 & 0.7111 & 10.0004 & 0.6465 \\
\hline & 6 & 0.6529 & 0.3787 & 0.7147 & 9.8782 & 0.6505 \\
\hline & 7 & 0.8061 & 0.5779 & 0.8720 & 8.7966 & 0.8015 \\
\hline & 8 & 0.8061 & 0.5779 & 0.8720 & 8.7966 & 0.8015 \\
\hline
\end{tabular}




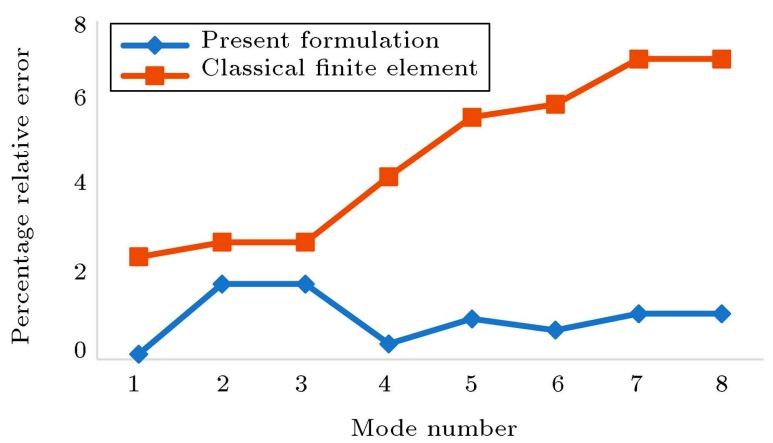

Figure 7. Error comparison for the fully clamped square plate with $t / a=0.1$.

As is seen in the tables, the present formulation is in quite better agreement with the analytical results, compared with the classical finite element. Moreover, Table 5 presents the first six natural frequencies in the case of SCSC. The first six natural frequency parameters were obtained in [28], and the results of the proposed formulation are compared with them. For clarity, Figure 7 shows the percentage of relative errors for a fully clamped square plate with $t / a=0.1$. In addition, Figure 8 depicts the first nine mode shapes of the simply supported square plate.

\subsection{Example 3: Free vibration of skew plate}

The third numerical example consists of a skew plate characterized by various skew angles and thicknesses. Results, which are obtained from the present formulation, are compared with the classical finite element and analytical solution ones. The geometry parameters for this example are shown in Figure 9. To compare the results, the natural frequency parameter is taken as follows [29]:

$$
\lambda=\frac{\omega b^{2}}{\pi^{2}} \sqrt{\frac{\rho t}{D}} .
$$

In this example, skew angles $\theta=15^{\circ}, \theta=30^{\circ}$, and $\theta=45^{\circ}$ with fully clamped and simply supported boundary conditions are considered. Moreover, all cases are solved by $6 \times 6$ meshes. Tables $6-11$ represent the results of simple and clamped boundary conditions

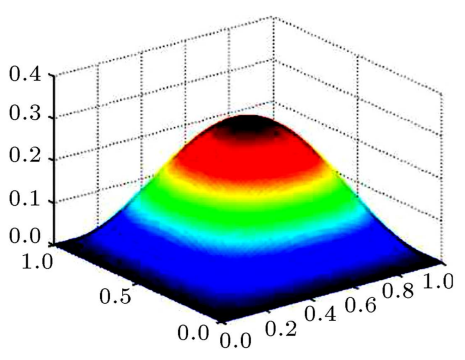

1 st

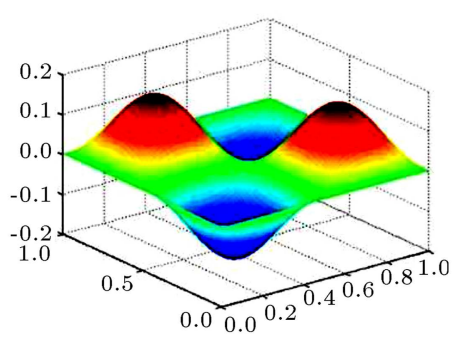

4 th

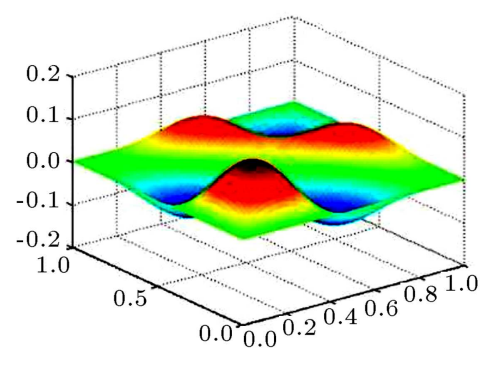

7 th

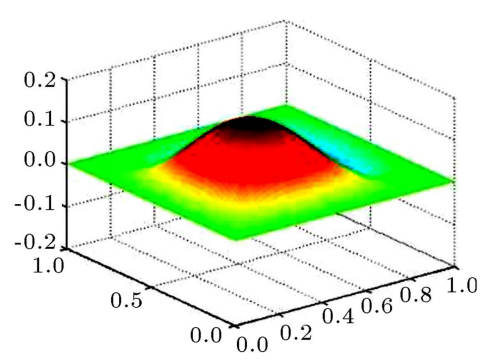

2nd

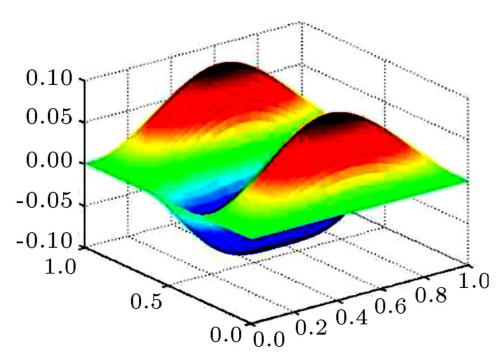

5 th

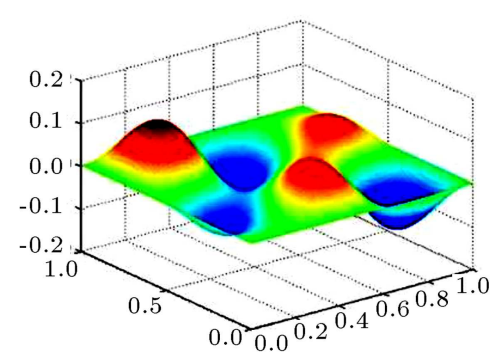

8 th

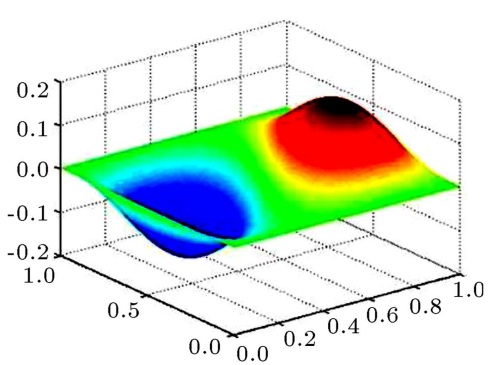

$3 r d$

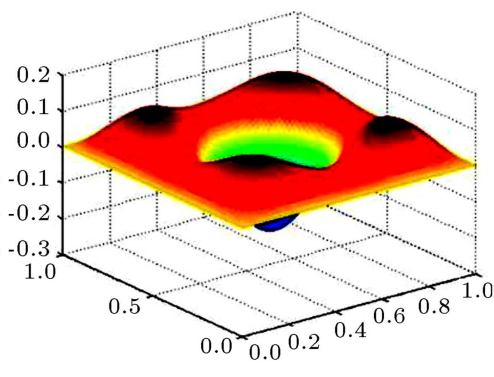

6 th

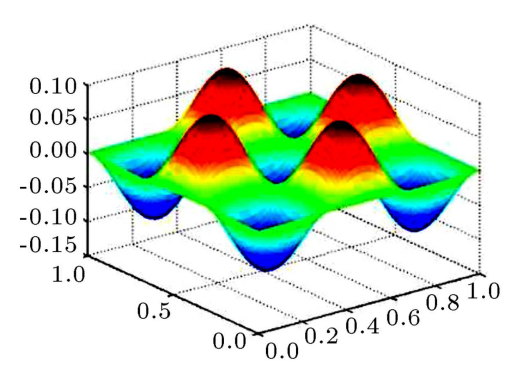

9 th

Figure 8. The first nine mode shapes of the simply supported square plate. 
Table 5. Dimensionless natural frequencies $\bar{\omega}$ of SCSC square case.

\begin{tabular}{ccccccc}
\hline $\boldsymbol{t} / \boldsymbol{a}$ & Mode no & $\begin{array}{c}\text { Present } \\
\text { formulation }\end{array}$ & Error (\%) & Classical FEM & Error (\%) & Analytical [28] \\
\hline & 1 & 1.2833 & 1.4303 & 1.2869 & 1.1570 & 1.302 \\
0.1 & 2.3664 & 1.3174 & 2.3873 & 0.4429 & 2.398 \\
& 2 & 2.8679 & 0.6955 & 2.9230 & 1.2138 & 2.888 \\
& 3 & 3.7812 & 1.8372 & 3.8385 & 0.3494 & 3.852 \\
& 5 & 4.2399 & 0.0704 & 4.4204 & 4.3301 & 4.237 \\
& 6 & 4.9795 & 0.8816 & 5.2988 & 7.3506 & 4.936 \\
& & & & & \\
& 1 & 0.1402 & 0.6014 & 0.1409 & 0.1066 & 0.1411 \\
& 2 & 0.2654 & 0.5240 & 0.2695 & 1.0367 & 0.2668 \\
& 3 & 0.3351 & 0.7441 & 0.3464 & 2.5943 & 0.3377 \\
& 4 & 0.4572 & 0.7664 & 0.4709 & 2.1973 & 0.4608 \\
& 5 & 0.4968 & 0.2043 & 0.5258 & 5.6215 & 0.4979 \\
& 6 & 0.6294 & 0.2432 & 0.6992 & 11.3565 & 0.6279 \\
\hline
\end{tabular}

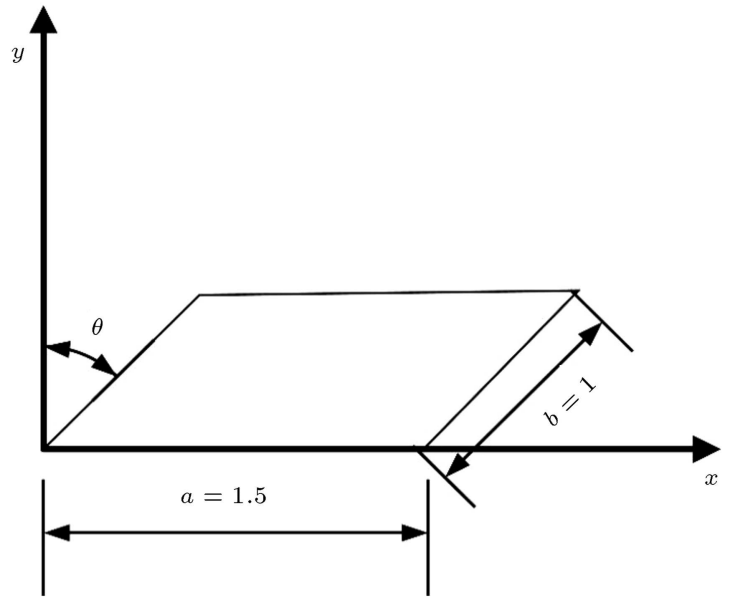

Figure 9. Geometry parameter for a skew plate.

with different skew angles. The application of spherical Hankel shape functions leads to better results than classic FEM, which is clearly shown in Tables 6-11. As results show, the frequency parameter increases by increasing the skew angle for all cases. To facilitate a greater understanding of the matter, Figure 10 depicts the first 4 mode shapes of fully clamped boundary conditions with $\theta=15^{\circ}$ and $t=0.1$. Generally, by decreasing the skew angle, the accuracy increases. However, at high skew angles, results are also acceptable.

\subsection{Example 4: Trapezoidal plate}

For the fourth numerical example, a trapezoidal plate with fully clamped and simply supported boundary conditions is investigated. Figure 11 demonstrates the geometry parameters of trapezoid. Tables 12-19 show

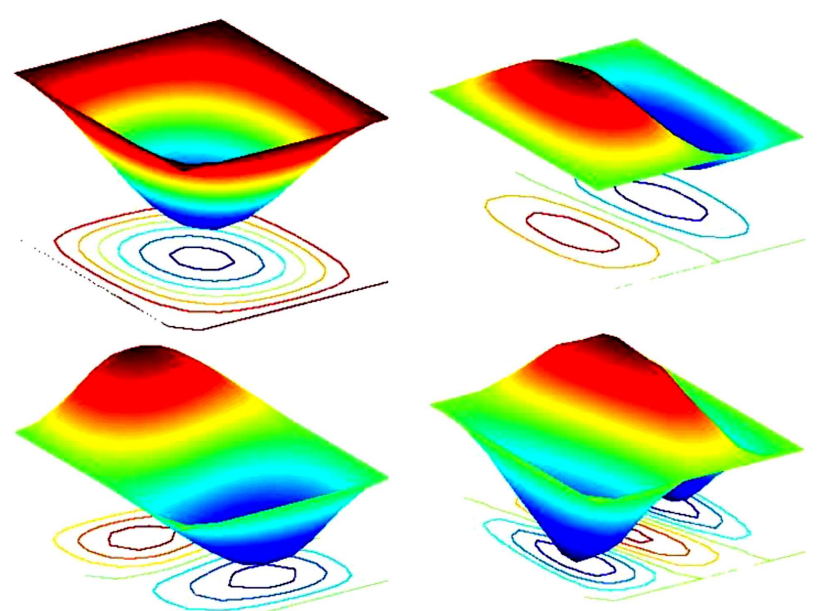

Figure 10. The first 4 mode shapes of the fully clamped skew plate $(\theta=15$ and $t=0.1)$.

the first six frequency parameters that are taken as [30]:

$$
\lambda=\frac{\omega a^{2}}{\pi^{2}} \sqrt{\frac{\rho t}{D}}
$$

For all cases, $6 \times 6$ meshes are employed. As expected, the frequency parameter for the clamped plate is considerably higher than that for the simply supported one. Thus, it can be concluded that by increasing restrictions in boundary conditions, the frequency parameter increases. In fact, the clamped plate is rigider than the simply supported one, which results in having a higher frequency parameter. Numerical results show that increasing the thickness-to-side ratio leads to a reduction in errors. Figure 12 shows the first nine mode shapes for clamped boundary conditions and 
Table 6. Frequency parameters of skew plate with simply supported boundary conditions $\left(\theta=15^{\circ}\right)$.

\begin{tabular}{|c|c|c|c|c|c|c|}
\hline Thickness & Mode no. & $\begin{array}{c}\text { Present } \\
\text { formulation }\end{array}$ & Error (\%) & Classical FEM & Error (\%) & Liew et al. [29] \\
\hline \multirow{8}{*}{0.1} & 1 & 1.5105 & 1.423 & 1.5181 & 1.936 & 1.4893 \\
\hline & 2 & 2.7795 & 1.126 & 2.812 & 2.305 & 2.7486 \\
\hline & 3 & 4.3695 & 0.644 & 4.4222 & 0.555 & 4.3978 \\
\hline & 4 & 4.6529 & 0.257 & 4.7519 & 2.389 & 4.641 \\
\hline & 5 & 5.6329 & 0.379 & 5.7195 & 1.152 & 5.6544 \\
\hline & 6 & 6.817 & 0.087 & 6.9888 & 2.611 & 6.811 \\
\hline & 7 & 7.8949 & 0.459 & 8.1415 & 3.597 & 7.8588 \\
\hline & 8 & 8.5893 & 0.841 & 8.7928 & 1.508 & 8.6622 \\
\hline \multirow{8}{*}{0.2} & 1 & 1.3953 & 0.628 & 1.4001 & 0.976 & 1.3866 \\
\hline & 2 & 2.4478 & 0.355 & 2.4682 & 1.194 & 2.4391 \\
\hline & 3 & 3.6913 & 0.395 & 3.7249 & 0.512 & 3.7059 \\
\hline & 4 & 3.8845 & 0.004 & 3.9454 & 1.573 & 3.8843 \\
\hline & 5 & 4.5969 & 0.259 & 4.6483 & 0.854 & 4.6089 \\
\hline & 6 & 5.4125 & 0.162 & 5.5124 & 2.012 & 5.4037 \\
\hline & 7 & 6.1315 & 0.536 & 6.2698 & 2.803 & 6.0988 \\
\hline & 8 & 6.5975 & 0.314 & 6.7118 & 1.413 & 6.6183 \\
\hline
\end{tabular}

Table 7. Frequency parameters of skew plate with simply supported boundary conditions $\left(\theta=30^{\circ}\right)$.

\begin{tabular}{|c|c|c|c|c|c|c|}
\hline Thickness & Mode no. & $\begin{array}{c}\text { Present } \\
\text { formulation }\end{array}$ & Error (\%) & Classical FEM & Error (\%) & Liew et al. [29] \\
\hline \multirow{8}{*}{0.1} & 1 & 1.858 & 4.282 & 1.8835 & 5.713 & 1.7817 \\
\hline & 2 & 3.2353 & 3.992 & 3.3281 & 6.975 & 3.1111 \\
\hline & 3 & 5.0198 & 1.2 & 5.2358 & 5.554 & 4.9603 \\
\hline & 4 & 5.2789 & 1.222 & 5.4058 & 1.153 & 5.3442 \\
\hline & 5 & 6.7841 & 0.636 & 7.0343 & 3.029 & 6.8275 \\
\hline & 6 & 7.0174 & 0.27 & 7.3831 & 4.927 & 7.0364 \\
\hline & 7 & 9.1685 & 0.574 & 9.7341 & 5.56 & 9.2214 \\
\hline & 8 & 9.169 & 0.745 & 9.7501 & 5.545 & 9.2378 \\
\hline \multirow{8}{*}{0.2} & 1 & 1.6746 & 2.181 & 1.6862 & 2.884 & 1.6389 \\
\hline & 2 & 2.7758 & 1.805 & 2.8178 & 3.343 & 2.7266 \\
\hline & 3 & 4.1333 & 0.426 & 4.2336 & 2.861 & 4.1158 \\
\hline & 4 & 4.3616 & 0.64 & 4.4231 & 0.761 & 4.3897 \\
\hline & 5 & 5.3941 & 0.361 & 5.5036 & 1.66 & 5.4137 \\
\hline & 6 & 5.5529 & 0.039 & 5.7176 & 2.925 & 5.5551 \\
\hline & 7 & 6.9814 & 0.125 & 7.2298 & 3.687 & 6.9727 \\
\hline & 8 & 6.9902 & 0.096 & 7.2374 & 3.636 & 6.9835 \\
\hline
\end{tabular}


Table 8. Frequency parameters of skew plate with simply supported boundary conditions $\left(\theta=45^{\circ}\right)$.

\begin{tabular}{|c|c|c|c|c|c|c|}
\hline Thickness & Mode no. & $\begin{array}{c}\text { Present } \\
\text { formulation }\end{array}$ & Error (\%) & Classical FEM & Error (\%) & Liew et al. [29] \\
\hline \multirow{8}{*}{0.1} & 1 & 2.6409 & 5.421 & 2.7026 & 7.885 & 2.5051 \\
\hline & 2 & 4.2309 & 6.054 & 4.4423 & 11.353 & 3.9894 \\
\hline & 3 & 6.0681 & 2.644 & 6.4897 & 9.775 & 5.9118 \\
\hline & 4 & 7.3551 & 1.533 & 7.6695 & 2.676 & 7.4696 \\
\hline & 5 & 8.1633 & 0.339 & 8.7927 & 7.345 & 8.1911 \\
\hline & 6 & 9.1317 & 1.201 & 9.7537 & 5.528 & 9.2427 \\
\hline & 7 & 10.2458 & 1.321 & 11.2101 & 7.966 & 10.383 \\
\hline & 8 & 11.6758 & 0.836 & 12.881 & 9.4 & 11.7742 \\
\hline \multirow{8}{*}{0.2} & 1 & 2.3045 & 2.887 & 2.3302 & 4.037 & 2.2398 \\
\hline & 2 & 3.4996 & 2.963 & 3.584 & 5.446 & 3.3989 \\
\hline & 3 & 4.8423 & 1.126 & 5.0146 & 4.725 & 4.7884 \\
\hline & 4 & 5.7921 & 0.809 & 5.9295 & 1.545 & 5.8393 \\
\hline & 5 & 6.3058 & 0.2 & 6.5514 & 3.687 & 6.3184 \\
\hline & 6 & 6.9389 & 0.572 & 7.1762 & 2.828 & 6.9788 \\
\hline & 7 & 7.672 & 0.309 & 8.0467 & 4.559 & 7.6958 \\
\hline & 8 & 8.5553 & 0.252 & 9.0113 & 5.596 & 8.5338 \\
\hline
\end{tabular}

Table 9. Frequency parameters of skew plate with fully clamped boundary conditions $\left(\theta=15^{\circ}\right)$.

\begin{tabular}{|c|c|c|c|c|c|c|}
\hline Thickness & Mode no. & $\begin{array}{c}\text { Present } \\
\text { formulation }\end{array}$ & Error (\%) & Classical FEM & Error (\%) & Liew et al. [29] \\
\hline \multirow{8}{*}{0.1} & 1 & 2.6588 & 0.466 & 2.6757 & 0.167 & 2.6712 \\
\hline & 2 & 3.9316 & 0.625 & 3.9751 & 0.474 & 3.9563 \\
\hline & 3 & 5.9275 & 0.205 & 6.0404 & 1.695 & 5.9397 \\
\hline & 4 & 6.0381 & 0.596 & 6.1166 & 0.697 & 6.0743 \\
\hline & 5 & 7.1734 & 0.518 & 7.2733 & 0.869 & 7.2107 \\
\hline & 6 & 8.2744 & 0.636 & 8.453 & 2.808 & 8.2221 \\
\hline & 7 & 9.352 & 1.02 & 9.5765 & 3.446 & 9.2575 \\
\hline & 8 & 10.4947 & 0.348 & 10.7125 & 2.431 & 10.4583 \\
\hline \multirow{8}{*}{0.2} & 1 & 2.2161 & 0.258 & 2.2283 & 0.29 & 2.2218 \\
\hline & 2 & 3.1594 & 0.409 & 3.1898 & 0.549 & 3.1724 \\
\hline & 3 & 4.5056 & 0.338 & 4.5523 & 0.694 & 4.5209 \\
\hline & 4 & 4.5215 & 0.169 & 4.5942 & 1.435 & 4.5292 \\
\hline & 5 & 5.2833 & 0.282 & 5.3431 & 0.847 & 5.2982 \\
\hline & 6 & 6.0092 & 0.404 & 6.1177 & 2.217 & 5.985 \\
\hline & 7 & 6.661 & 0.719 & 6.7941 & 2.732 & 6.6134 \\
\hline & 8 & 7.2374 & 0.05 & 7.3542 & 1.664 & 7.2338 \\
\hline
\end{tabular}


Table 10. Frequency parameters of skew plate with fully clamped boundary conditions $\left(\theta=30^{\circ}\right)$.

\begin{tabular}{|c|c|c|c|c|c|c|}
\hline Thickness & Mode no. & $\begin{array}{c}\text { Present } \\
\text { formulation }\end{array}$ & Error (\%) & Classical FEM & Error (\%) & Liew et al. [29] \\
\hline \multirow{8}{*}{0.1} & 1 & 3.1817 & 0.488 & 3.2037 & 0.201 & 3.1973 \\
\hline & 2 & 4.4981 & 0.665 & 4.5542 & 0.573 & 4.5282 \\
\hline & 3 & 6.4558 & 0.263 & 6.5882 & 1.783 & 6.4728 \\
\hline & 4 & 7.2251 & 0.568 & 7.3252 & 0.809 & 7.2664 \\
\hline & 5 & 8.5482 & 0.27 & 8.6881 & 1.362 & 8.5714 \\
\hline & 6 & 8.7001 & 0.662 & 8.9092 & 3.081 & 8.6429 \\
\hline & 7 & 10.9597 & 1.464 & 11.2495 & 4.147 & 10.8016 \\
\hline & 8 & 11.071 & 1.85 & 11.3741 & 4.638 & 10.8699 \\
\hline \multirow{8}{*}{0.2} & 1 & 2.5823 & 0.244 & 2.5973 & 0.335 & 2.5886 \\
\hline & 2 & 3.5278 & 0.433 & 3.5654 & 0.625 & 3.5432 \\
\hline & 3 & 4.8265 & 0.221 & 4.9086 & 1.477 & 4.8372 \\
\hline & 4 & 5.2184 & 0.299 & 5.2741 & 0.766 & 5.234 \\
\hline & 5 & 6.096 & 0.125 & 6.1761 & 1.188 & 6.1036 \\
\hline & 6 & 6.2255 & 0.396 & 6.3499 & 2.403 & 6.2009 \\
\hline & 7 & 7.5553 & 0.965 & 7.7193 & 3.156 & 7.4831 \\
\hline & 8 & 7.643 & 1.207 & 7.8183 & 3.527 & 7.5519 \\
\hline
\end{tabular}

Table 11. Frequency parameters of the skew plate with fully clamped boundary conditions $\left(\theta=45^{\circ}\right)$.

\begin{tabular}{|c|c|c|c|c|c|c|}
\hline Thickness & Mode no. & $\begin{array}{c}\text { Present } \\
\text { formulation }\end{array}$ & Error (\%) & Classical FEM & Error (\%) & Liew et al. [29] \\
\hline \multirow{8}{*}{0.1} & 1 & 4.4218 & 0.599 & 4.4607 & 0.276 & 4.4484 \\
\hline & 2 & 5.8102 & 0.869 & 5.9071 & 0.783 & 5.8612 \\
\hline & 3 & 7.816 & 0.442 & 8.0251 & 2.222 & 7.8507 \\
\hline & 4 & 9.7049 & 0 & 9.9273 & 2.291 & 9.7049 \\
\hline & 5 & 10.3707 & 0.022 & 10.6303 & 2.526 & 10.3684 \\
\hline & 6 & 11.375 & 0.437 & 11.6411 & 2.787 & 11.3255 \\
\hline & 7 & 12.621 & 1.573 & 13.0527 & 5.048 & 12.4255 \\
\hline & 8 & 14.0466 & 2.771 & 14.5349 & 6.344 & 13.6679 \\
\hline \multirow{8}{*}{0.2} & 1 & 3.3958 & 0.255 & 3.4185 & 0.412 & 3.4045 \\
\hline & 2 & 4.3408 & 0.535 & 4.3982 & 0.78 & 4.3642 \\
\hline & 3 & 5.61 & 0.334 & 5.7272 & 1.748 & 5.6288 \\
\hline & 4 & 6.6673 & 0.114 & 6.7683 & 1.399 & 6.6749 \\
\hline & 5 & 7.0803 & 0.207 & 7.2321 & 2.356 & 7.0657 \\
\hline & 6 & 7.6736 & 0.249 & 7.8091 & 2.019 & 7.6546 \\
\hline & 7 & 8.4295 & 1.113 & 8.6623 & 3.905 & 8.3367 \\
\hline & 8 & 9.2019 & 1.752 & 9.454 & 4.539 & 9.0435 \\
\hline
\end{tabular}




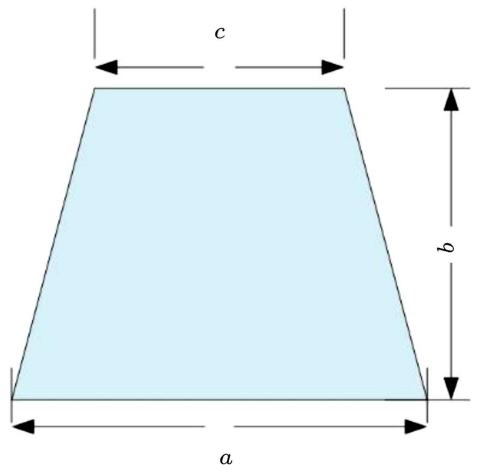

Figure 11. Trapezoid's geometry parameters.

$c / a=4 / 5, b / a=1$, and $t / a=0.2$. According to the results, the present formulation is much more efficient than the classical FEM.

\subsection{Example 5: Buckling of rectangular plate}

The last numerical example consists of a square plate $(a=1)$ with different boundary conditions and loadings. The mesh here is considered coarse $(2 \times 2)$ to show that with lower degrees of freedom, high accuracy is reachable using spherical Hankel shape functions. In order to compare results with those available in the literature, buckling factor is defined as $k=\frac{F_{c r}}{\pi^{2} D}$ in which $F_{c r}$ is the critical force [31]. Figure 13 lists the geometry parameters, loadings, and boundary conditions for this example.
Table 20 lists the buckling factors for the square plate obtained through the present formulation, classical finite element, and analytical one. It can be observed that the results of the spherical Hankel shape functions provide excellent agreement with those obtained by analytical solutions, compared to the Lagrange shape functions. For example, in the case of $\operatorname{SCSC}(h / a=0.05)$, the percentage of error in the proposed method is almost zero, while it is $1.67 \%$ in the classic Lagrange shape functions. In addition, Figure 14 illustrates the first 4 buckling modes in the case of SSSF and $t / a=0.05$.

To compare CPU time of the present formulation and Lagrangian FEM, the consumed time for the case $t / a=0.001$ with different boundary conditions is shown in Figure 15. According to this figure, it is obvious that the application of Hankel shape functions results in greater time consumption. It is worthwhile to note that Hankel shape functions contain polynomial terms and functional expansion; therefore, it is reasonable to assume that Hankel shape functions are computationally a bit more expensive than the classic FEM. Nonetheless, the accuracy and robustness provided by Hankel shape functions encourage their use.

According to the literature, shape parameters are constants used in RBFs to increase the accuracy [8-24]. It can be said that any specific problem requires the

Table 12. Frequency parameters of trapezoidal plate with simply supported boundary conditions $(c / a=3 / 5$ and $b / a=1)$.

\begin{tabular}{|c|c|c|c|c|c|c|}
\hline$t / a$ & $\begin{array}{c}\text { Mode } \\
\text { sequence }\end{array}$ & $\begin{array}{c}\text { Present } \\
\text { formulation }\end{array}$ & Error (\%) & Classical FEM & Error (\%) & Analytical [30] \\
\hline \multirow{6}{*}{0.1} & 1 & 3.9620 & 5.7585 & 3.9963 & 6.6740 & 3.7463 \\
\hline & 2 & 8.0375 & 2.3469 & 8.1880 & 4.2638 & 7.8532 \\
\hline & 3 & 9.8812 & 1.2137 & 10.0958 & 3.4117 & 9.7628 \\
\hline & 4 & 13.9380 & 0.0548 & 14.4793 & 3.8266 & 13.9457 \\
\hline & 5 & 14.1527 & 0.9576 & 14.4915 & 3.3742 & 14.0185 \\
\hline & 6 & 17.6804 & 0.7921 & 18.3975 & 3.2314 & 17.8216 \\
\hline \multirow{6}{*}{0.2} & 1 & 3.5058 & 6.6036 & 3.5347 & 7.4818 & 3.2887 \\
\hline & 2 & 6.6093 & 2.9470 & 6.74077 & 4.9949 & 6.4201 \\
\hline & 3 & 7.8737 & 1.1652 & 8.04974 & 3.4259 & 7.7831 \\
\hline & 4 & 10.5365 & 0.1815 & 10.9468 & 4.0828 & 10.5174 \\
\hline & 5 & 10.6840 & 0.7431 & 10.9625 & 3.3695 & 10.6052 \\
\hline & 6 & 12.8179 & 1.1328 & 13.3497 & 2.9689 & 12.9648 \\
\hline
\end{tabular}


Table 13. Frequency parameters of trapezoidal plate with SSSS boundary conditions $(c / a=3 / 5$ and $b / a=2)$.

\begin{tabular}{ccccccc}
\hline $\boldsymbol{t} / \boldsymbol{a}$ & $\begin{array}{c}\text { Mode } \\
\text { sequence }\end{array}$ & $\begin{array}{c}\text { Present } \\
\text { formulation }\end{array}$ & Error (\%) & Classical FEM & Error (\%) & Analytical [30] \\
\hline & 1 & 2.6896 & 2.1548 & 2.7145 & 3.0997 & 2.6329 \\
& 2 & 3.9739 & 2.2139 & 4.0364 & 3.8218 & 3.8879 \\
0.1 & 3 & 5.6679 & 1.2144 & 5.8527 & 4.5146 & 5.5999 \\
& 4 & 7.9330 & 0.1756 & 8.2864 & 4.6384 & 7.9191 \\
& 5 & 8.0812 & 0.5570 & 8.3905 & 3.2492 & 8.1265 \\
& 6 & 10.1363 & 0.5482 & 10.4312 & 2.3456 & 10.1922 \\
& & & & & 3.7501 & 2.4001 \\
& 1 & 2.4640 & 2.6625 & 2.4901 & 3.4182 \\
& 2 & 3.5300 & 3.2731 & 3.5965 & 5.2189 & 4.7521 \\
& 3 & 4.8410 & 1.8720 & 5.0289 & 5.8266 & 6.479 \\
& 4 & 6.4640 & 0.2301 & 6.8173 & 5.2219 & 6.666 \\
\hline
\end{tabular}

Table 14. Frequency parameters of trapezoidal plate with SSSS boundary conditions $(c / a=4 / 5$ and $b / a=1)$.

\begin{tabular}{ccccccc}
\hline $\boldsymbol{t} / \boldsymbol{a}$ & $\begin{array}{c}\text { Mode } \\
\text { sequence }\end{array}$ & $\begin{array}{c}\text { Present } \\
\text { formulation }\end{array}$ & Error (\%) & Classical FEM & Error (\%) & Analytical [30] \\
\hline & 1 & 3.3819 & 4.0440 & 3.4077 & 4.8388 & 3.2505 \\
& 2 & 7.4434 & 1.0915 & 7.5979 & 3.1901 & 7.3631 \\
0.1 & 3 & 8.3277 & 0.4857 & 8.5116 & 2.7050 & 8.2875 \\
& 4 & 12.1057 & 1.4459 & 12.4034 & 3.9408 & 11.9332 \\
& 5 & 13.4090 & 1.5408 & 13.9861 & 2.6965 & 13.6189 \\
& 6 & 15.3369 & 1.7642 & 16.0086 & 2.5382 & 15.6124 \\
& & & & & \\
& 1 & 3.0614 & 6.2428 & 3.0819 & 6.9538 & 2.8816 \\
& 2 & 6.2177 & 2.2965 & 6.3307 & 4.1552 & 6.0782 \\
& 3 & 6.8482 & 1.3427 & 6.9786 & 3.2722 & 6.7575 \\
& 4 & 9.4320 & 2.4635 & 9.6287 & 4.6001 & 9.2053 \\
& 5 & 10.2807 & 0.7813 & 10.6560 & 2.8405 & 10.3617 \\
& 5 & 11.4956 & 1.1207 & 11.9186 & 2.5180 & 11.6259 \\
\hline
\end{tabular}

most suitable shape parameter by its own nature, provided that high accuracy is demanded. In the last part of the paper, some shape parameters, used in numerical examples, are summarized in Table 21. It is worthwhile to note that the shape parameters are inseparable from RBFs. By means of illustration, conical, multiquadric, inverse multiquadric, Gaussian, and J-Bessel functions [12] have only one shape parameter, whilst complex Fourier [11] and Hankel RBFs [21-23] have two of them. For the Hankel shape functions, $\tilde{n}$ and $e$ are the shape parameters that belong to the set of whole numbers and positive real numbers, respectively. 
Table 15. Frequency parameters of trapezoidal plate with SSSS boundary conditions $(c / a=4 / 5$ and $b / a=2)$.

\begin{tabular}{ccccccc}
\hline $\boldsymbol{t} / \boldsymbol{a}$ & $\begin{array}{c}\text { Mode } \\
\text { sequence }\end{array}$ & $\begin{array}{c}\text { Present } \\
\text { formulation }\end{array}$ & Error (\%) & Classical FEM & Error (\%) & Analytical [30] \\
\hline & 1 & 2.2543 & 1.4600 & 2.2714 & 2.2316 & 2.2219 \\
& 2 & 3.3603 & 1.9477 & 3.4129 & 3.5436 & 3.2961 \\
0.1 & 3 & 5.0579 & 0.4055 & 5.2373 & 3.9678 & 5.0375 \\
& 4 & 7.1438 & 1.1642 & 7.3181 & 1.2468 & 7.228 \\
& 5 & 7.3580 & 0.7001 & 7.8156 & 5.4759 & 7.4099 \\
& 6 & 8.4117 & 0.6521 & 8.6284 & 1.9068 & 8.467 \\
& & & & & & 2.0471 \\
& 1 & 2.1019 & 2.6788 & 2.1165 & 3.3942 & 2.9311 \\
& 2 & 3.0485 & 4.0068 & 3.0927 & 5.5135 & 4.3287 \\
& 3 & 4.4185 & 2.0761 & 4.5610 & 5.3674 & 6.0327 \\
& 5.2 & 5.9987 & 0.5631 & 6.1271 & 1.5651 & 6.1265 \\
& 4 & 6.1511 & 0.4026 & 6.4906 & 5.9435 & 6.9002 \\
\hline
\end{tabular}

Table 16. Frequency parameters of trapezoidal plate with CCCC boundary conditions $(c / a=3 / 5$ and $b / a=1)$.

\begin{tabular}{|c|c|c|c|c|c|c|}
\hline$t / a$ & $\begin{array}{c}\text { Mode } \\
\text { sequence }\end{array}$ & $\begin{array}{c}\text { Present } \\
\text { formulation }\end{array}$ & Error (\%) & Classical FEM & Error (\%) & Analytical [30] \\
\hline \multirow{6}{*}{0.1} & 1 & 6.6142 & 0.3623 & 6.6431 & 0.07243 & 6.6383 \\
\hline & 2 & 11.0119 & 0.4054 & 11.0974 & 0.3677 & 11.0568 \\
\hline & 3 & 13.2786 & 0.4091 & 13.3932 & 0.4503 & 13.3332 \\
\hline & 4 & 17.3058 & 0.1997 & 17.5354 & 1.5287 & 17.2714 \\
\hline & 5 & 17.5712 & 0.3726 & 17.7254 & 0.5016 & 17.637 \\
\hline & 6 & 21.5327 & 0.1964 & 21.8193 & 1.5302 & 21.4905 \\
\hline \multirow{6}{*}{0.2} & 1 & 5.1827 & 0.2471 & 5.1981 & 0.0495 & 5.1956 \\
\hline & 2 & 8.0816 & 0.2679 & 8.1239 & 0.2532 & 8.1034 \\
\hline & 3 & 9.3634 & 0.266 & 9.4155 & 0.2887 & 9.3884 \\
\hline & 4 & 11.8942 & 0.1439 & 12 & 1.0339 & 11.8772 \\
\hline & 5 & 11.9785 & 0.2388 & 12.047 & 0.3314 & 12.0072 \\
\hline & 6 & 14.1851 & 0.1434 & 14.3102 & 1.0270 & 14.1648 \\
\hline
\end{tabular}

\section{Conclusion}

In this research, a new class of shape functions called spherical Hankel shape function was introduced. These shape functions were applied to reformulate deflection, free vibration, and buckling of Mindlin plates theory based on Finite Element Method (FEM). These new shape functions include the advantages of both radial basis functions and spherical Bessel functions, which in turn result in greater robustness and strength for the 
Table 17. Frequency parameters of trapezoidal plate with CCCC boundary conditions $(c / a=3 / 5$ and $b / a=2)$.

\begin{tabular}{ccccccc}
\hline $\boldsymbol{t} / \boldsymbol{a}$ & $\begin{array}{c}\text { Mode } \\
\text { sequence }\end{array}$ & $\begin{array}{c}\text { Present } \\
\text { formulation }\end{array}$ & Error (\%) & Classical FEM & Error (\%) & Analytical [30] \\
\hline & 1 & 4.9327 & 1.0924 & 4.9936 & 0.1295 & 4.9872 \\
& 2 & 6.3178 & 1.1644 & 6.4134 & 0.3315 & 6.3923 \\
& 3 & 7.9211 & 1.1809 & 8.1020 & 1.0758 & 8.0158 \\
& 4 & 10.1535 & 0.2925 & 10.532 & 3.4242 & 10.1833 \\
& 5 & 11.0411 & 1.7098 & 11.2981 & 0.5782 & 11.2332 \\
& 6 & 13.1888 & 2.1034 & 13.5084 & 4.5777 & 12.9171 \\
& & & & & \\
& 1 & 4.0129 & 0.43929 & 4.0344 & 0.0926 & 4.0307 \\
& 2 & 4.9979 & 0.4243 & 5.0314 & 0.2445 & 5.0192 \\
& 3 & 6.1700 & 0.2234 & 6.2380 & 0.8751 & 6.1839 \\
& 4 & 7.7625 & 0.9030 & 7.9030 & 2.7289 & 7.6931 \\
& 4 & 8.0906 & 0.5696 & 8.1679 & 0.3801 & 8.137 \\
& 5 & 9.4278 & 0.4399 & 9.5224 & 0.5589 & 9.4695 \\
\hline
\end{tabular}

Table 18. Frequency parameters of trapezoidal plate with CCCC boundary conditions $(c / a=4 / 5$ and $b / a=1)$.

\begin{tabular}{|c|c|c|c|c|c|c|}
\hline$t / a$ & $\begin{array}{c}\text { Mode } \\
\text { sequence }\end{array}$ & $\begin{array}{c}\text { Present } \\
\text { formulation }\end{array}$ & Error (\%) & Classical FEM & Error (\%) & Analytical [30] \\
\hline \multirow{6}{*}{0.1} & 1 & 5.7375 & 0.3789 & 5.7631 & 0.0648 & 5.7594 \\
\hline & 2 & 10.2571 & 0.4363 & 10.3439 & 0.4064 & 10.3021 \\
\hline & 3 & 11.3903 & 0.4329 & 11.4908 & 0.4450 & 11.4399 \\
\hline & 4 & 15.2196 & 0.4514 & 15.3562 & 0.4416 & 15.2887 \\
\hline & 5 & 16.7366 & 0.2117 & 16.9724 & 1.6237 & 16.7013 \\
\hline & 6 & 18.9659 & 0.2160 & 19.2318 & 1.6206 & 18.9251 \\
\hline \multirow{6}{*}{0.2} & 1 & 4.6084 & 0.2748 & 4.6233 & 0.0468 & 4.6212 \\
\hline & 2 & 7.6185 & 0.3095 & 7.6633 & 0.2763 & 7.6422 \\
\hline & 3 & 8.2736 & 0.3054 & 8.3231 & 0.2910 & 8.299 \\
\hline & 4 & 10.6932 & 0.3070 & 10.7586 & 0.3023 & 10.7262 \\
\hline & 5 & 11.5552 & 0.1194 & 11.6675 & 1.0919 & 11.5415 \\
\hline & 6 & 12.7817 & 0.1203 & 12.904 & 1.0818 & 12.7664 \\
\hline
\end{tabular}

offered formulations. Moreover, new shape functions can simply satisfy spherical Bessel function fields in addition to polynomial functions. Due to their properties, achieving higher accuracy with lower degrees of freedom is accessible. Therefore, to gain highly accu- rate results through FEM, instead of using fine meshes, the proposed shape function with coarse meshes can be utilized. It should be noted that through lower degrees of freedom, computational cost was reduced. Finally, in order to show the precision and reliability of the offered 
Table 19. Frequency parameters of trapezoidal plate with CCCC boundary conditions $(c / a=4 / 5$ and $b / a=2)$.

\begin{tabular}{|c|c|c|c|c|c|c|}
\hline$t / a$ & $\begin{array}{c}\text { Mode } \\
\text { sequence }\end{array}$ & $\begin{array}{c}\text { Present } \\
\text { formulation }\end{array}$ & Error (\%) & Classical FEM & Error (\%) & Analytical [30] \\
\hline \multirow{6}{*}{0.1} & 1 & 4.2583 & 0.5661 & 4.2863 & 0.0875 & 4.2826 \\
\hline & 2 & 5.2811 & 0.6470 & 5.3273 & 0.2222 & 5.3155 \\
\hline & 3 & 6.9461 & 0.4005 & 7.0551 & 1.1623 & 6.9741 \\
\hline & 4 & 9.4191 & 1.32346 & 9.6581 & 3.8947 & 9.2961 \\
\hline & 5 & 10.0170 & 0.7919 & 10.1514 & 0.5395 & 10.097 \\
\hline & 6 & 11.2870 & 0.7558 & 11.4383 & 0.5743 & 11.373 \\
\hline \multirow{6}{*}{0.2} & 1 & 3.5169 & 0.4520 & 3.5351 & 0.0631 & 3.5329 \\
\hline & 2 & 4.2913 & 0.5317 & 4.3222 & 0.18431 & 4.3143 \\
\hline & 3 & 5.5234 & 0.3645 & 5.5968 & 0.9588 & 5.5437 \\
\hline & 4 & 7.2340 & 0.9148 & 7.3869 & 3.0477 & 7.1685 \\
\hline & 5 & 7.3936 & 0.6217 & 7.4663 & 0.3558 & 7.4399 \\
\hline & 6 & 8.2143 & 0.5966 & 8.2951 & 0.3804 & 8.2637 \\
\hline
\end{tabular}

Table 20. Buckling factor of square plate with various boundary conditions.

\begin{tabular}{clcccc}
\hline \multirow{2}{*}{$\begin{array}{c}\text { Boundary } \\
\text { condition }\end{array}$} & Method & \multicolumn{5}{c}{$\boldsymbol{t} / \mathbf{a}$} \\
\cline { 3 - 6 } & & $\mathbf{0 . 0 0 1}$ & $\mathbf{0 . 0 5}$ & $\mathbf{0 . 1}$ & $\mathbf{0 . 2}$ \\
\hline \multirow{2}{*}{ SSSF } & Classical FEM & 1.4165 & 1.3993 & 1.3556 & 1.2224 \\
& Present formulation & 1.4014 & 1.3812 & 1.3706 & 1.2137 \\
& Analytical [31] & 1.4014 & 1.3813 & 1.3707 & 1.2138 \\
& & & & & \\
\multirow{2}{*}{ SSSS } & Classical FEM & 4.0601 & 4.0578 & 3.8259 & 3.2656 \\
& Present formulation & 3.9999 & 3.9439 & 3.7845 & 3.2637 \\
& Analytical [31] & 4 & 3.9444 & 3.7846 & 3.2637 \\
& & & & & \\
& & & & & \\
& Classical FEM & 7.4811 & 7.1765 & 6.4186 & 4.5843 \\
& Present formulation & 7.6910 & 7.2989 & 6.3697 & 4.3203 \\
& Analytical [31] & 7.6911 & 7.2989 & 6.3698 & 4.3204 \\
\hline
\end{tabular}

Table 21. Shape parameter values used in examples.

\begin{tabular}{cccccc}
\hline & Example 1 & Example 2 & Example 3 & Example 4 & Example 5 \\
\hline$\tilde{n}$ & 2 & 3 & 3 & 4 & 6 \\
$e$ & 2.6189 & 2.7610 & 3.1425 & 1.1714 & 1.0631 \\
\hline
\end{tabular}



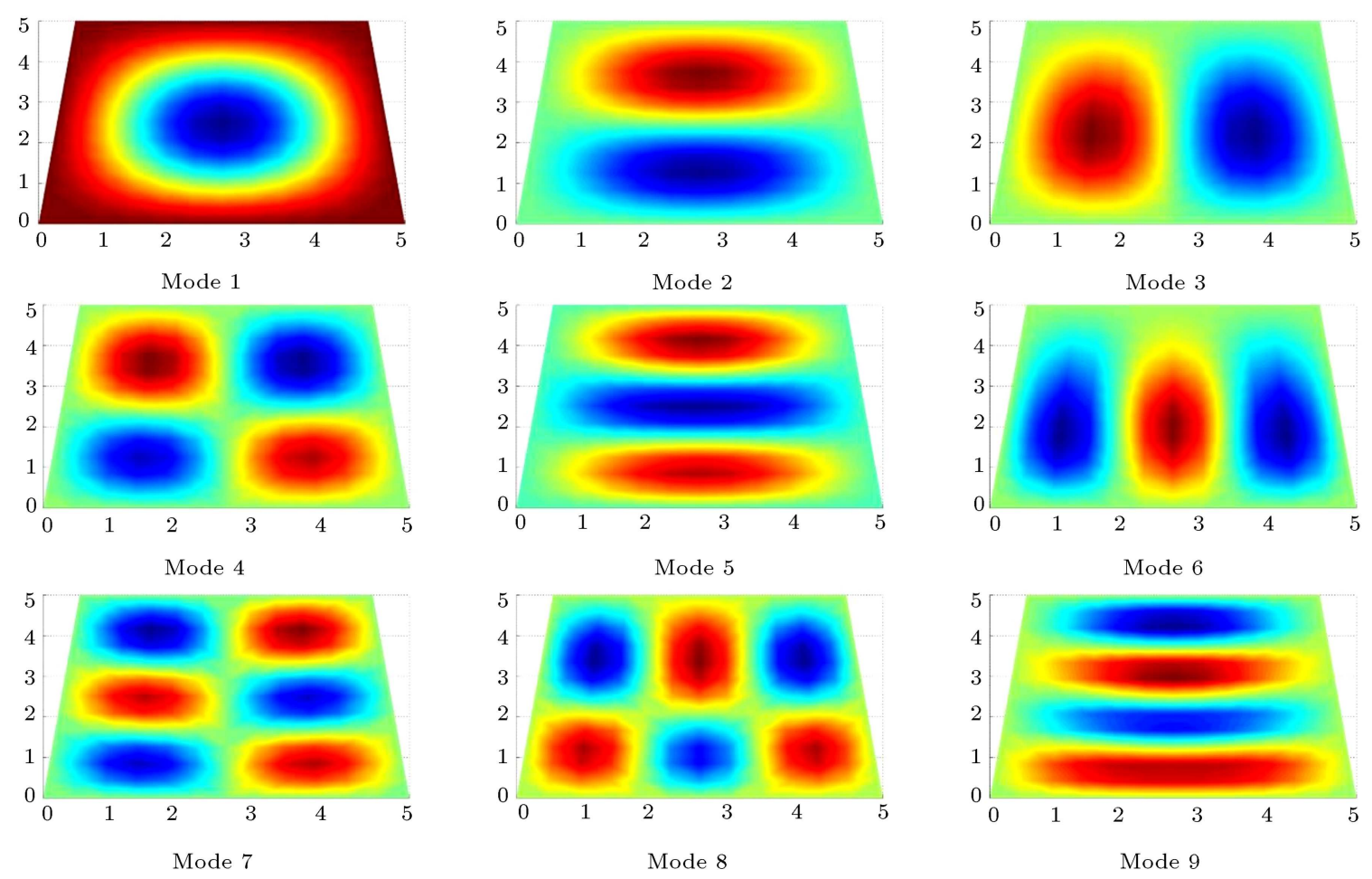

Figure 12. The first nine mode shapes for clamped boundary conditions.
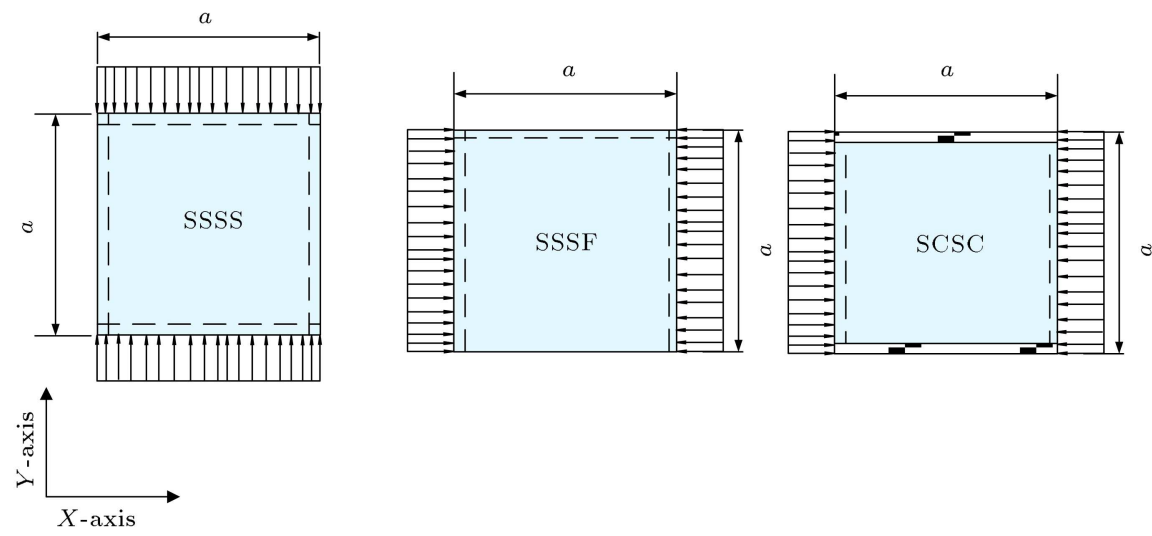

Figure 13. Geometry parameters, loadings, and boundary conditions of Example 5.
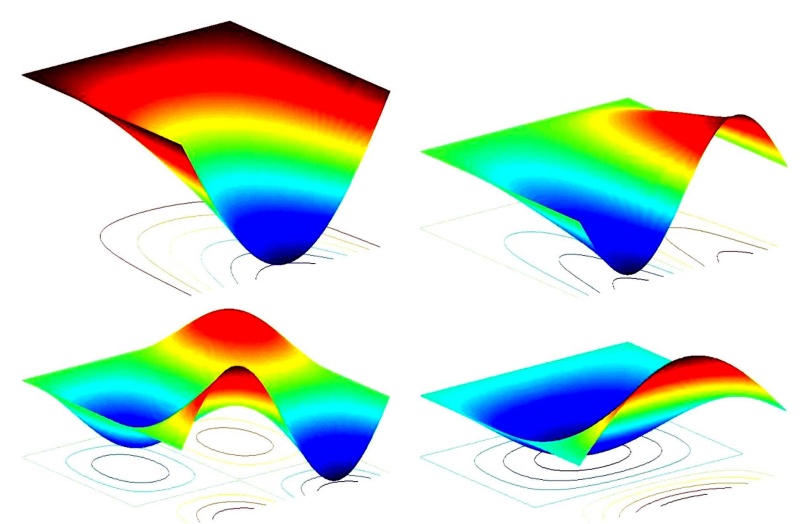

Figure 14. The first 4 buckling modes for the case of $\mathrm{SSSF}$ and $t / a=0.05$.

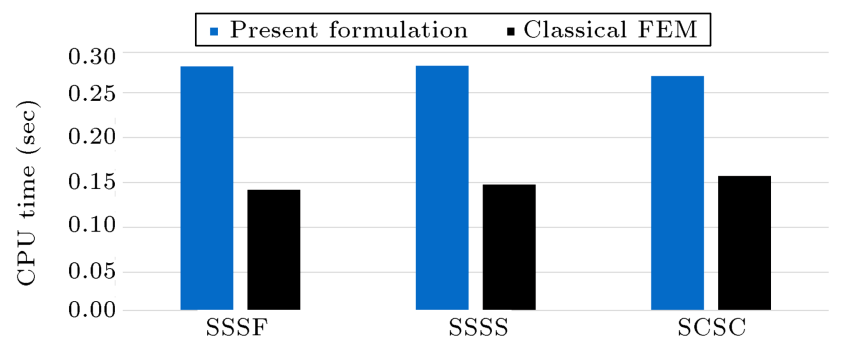

Figure 15. The elapsed time for case $t / a=0.001$.

approaches, five numerical examples were solved, and the achieved results were compared with the classical finite element and analytical results. According to the results, the suggested approaches are much more versatile than classical FEM. 


\section{Acknowledgements}

The authors would like to acknowledge and express their special gratitude to anonymous reviewers for their constructive pieces of advice that improved the manuscript.

\section{References}

1. Timoshenko, S. and Woinowsky-Krieger, S. Theory of Plates and Shells, McGraw-hill (1959).

2. Hughes, T.J. and Tezduyar, T. "Finite elements based upon Mindlin plate theory with particular reference to the four-node bilinear isoparametric element", Journal of Applied Mechanics, 48(3) pp. 587-596 (1981).

3. Tanaka, M. and Herein, A. "A boundary element method applied to the elastic bending problem of stiffened plates", WIT Transactions on Modelling and Simulation, 19, pp. 203-212 (1970).

4. Qian, L., Batra, R., and Chen, L. "Static and dynamic deformations of thick functionally graded elastic plates by using higher-order shear and normal deformable plate theory and meshless local PetrovGalerkin method", Composites Part B: Engineering, 35(6), pp. 685-697 (2004).

5. Chu, F., Wang, L., Zhong, Z., and He, J. "Hermite radial basis collocation method for vibration of functionally graded plates with in-plane material inhomogeneity", Computers \& Structures, 142, pp. 79-89 (2014).

6. Chu, F., He, J., Wang, L., and Zhong, Z. "Buckling analysis of functionally graded thin plate with in-plane material inhomogeneity", Engineering Analysis with Boundary Elements, 65, pp. 112-125 (2016).

7. Long, S. and Atluri, S. "A meshless local PetrovGalerkin method for solving the bending problem of a thin plate", Computer Modeling in Engineering and Sciences, 3(1), pp. 53-64 (2002).

8. Buhmann, M.D., Radial Basis Functions: Theory and Implementations. Vol. 12. Cambridge University Press (2003).

9. Hamzehei Javaran, S. and Khaji, N. "Dynamic analysis of plane elasticity with new complex Fourier radial basis functions in the dual reciprocity boundary element method", Applied Mathematical Modelling, 38(14), pp. 3641-3651 (2014).

10. Hamzeh Javaran, S., Khaji, N., and Moharrami, H. "A dual reciprocity BEM approach using new Fourier radial basis functions applied to $2 \mathrm{D}$ elastodynamic transient analysis", Engineering Analysis with Boundary Elements, 35(1), pp. 85-95 (2011).

11. Khaji, N. and Hamzehei Javaran, S. "New complex Fourier shape functions for the analysis of twodimensional potential problems using boundary element method", Engineering Analysis with Boundary Elements, 37(2), pp. 260-272 (2013).
12. Hamzeh Javaran, S., Khaji, N., and Noorzad, A. "First kind Bessel function (J-Bessel) as radial basis function for plane dynamic analysis using dual reciprocity boundary element method", Acta Mechanica, 218(34), pp. 247-258 (2011).

13. Rashed, Y.F. "Transient dynamic boundary element analysis using Gaussian-based mass matrix", Engineering Analysis with Boundary Elements, 26(3), pp. 265-279 (2002).

14. Agnantiaris, J., Polyzos, D., and Beskos, D. "Some studies on dual reciprocity BEM for elastodynamic analysis", Computational Mechanics, 17(4), pp. 270277 (1996).

15. Chen, C. "The method of fundamental solutions for non-linear thermal explosions", International Journal for Numerical Methods in Biomedical Engineering, 11(8), pp. 675-681 (1995).

16. Rashed, Y.F. "BEM for dynamic analysis using compact supported radial basis functions", Computers \& Structures, 80(16), pp. 1351-1367 (2002).

17. Samaan, M.F., Rashed, Y.F., and Ahmed, M.A. "The dual reciprocity method applied to free vibrations of 2D structures using compact supported radial basis functions", Computational Mechanics, 41(1), pp. 85105 (2007).

18. Wang, L. "Radial basis functions methods for boundary value problems: Performance comparison", Engineering Analysis with Boundary Elements, 84, pp. 191-205 (2017).

19. Samaan, M.F. and Rashed, Y.F. "Free vibration multiquadric boundary elements applied to plane elasticity", Applied Mathematical Modelling, 33(5), pp. 2421-2432 (2009).

20. Hamzehei Javaran, S. and Khaji, N. "Inverse multiquadric (IMQ) function as radial basis function for plane dynamic analysis using dual reciprocity boundary element method", 15th World Conference on Earthquake Engineering, Lisboa, Portugal (2012).

21. Hamzehei Javaran, S. and Shojaee, S. "The solution of elasto static and dynamic problems using the boundary element method based on spherical Hankel element framework", International Journal for Numerical Methods in Engineering, 112(13), pp. 2067-2086 (2017).

22. Farmani, S., Ghaeini-Hessaroeyeh, M., and Hamzehei Javaran, S. "The improvement of numerical modeling in the solution of incompressible viscous flow problems using finite element method based on spherical Hankel shape functions", International Journal for Numerical Methods in Fluids, 87(2), pp. 70-89 (2018).

23. Hamzehei-Javaran, S. and Shojaee, S. "Improvement of numerical modeling in the solution of static 
and transient dynamic problems using finite element method based on spherical Hankel shape functions", International Journal for Numerical Methods in Engineering, 115(10), pp. 1241-1265 (2018). DOI: $10.1002 /$ nme.5842)

24. Wang, J. and Liu, G. "On the optimal shape parameters of radial basis functions used for 2-D meshless methods", Computer Methods in Applied Mechanics and Engineering, 191(23), pp. 2611-2630 (2002).

25. Bhatti, M.A., Advanced Topics in Finite Element Analysis of Structures: with Mathematica and MAT$L A B$ Computations, John Wiley \& Sons, Inc. (2006).

26. Ferreira, A.J., MATLAB Codes for Finite Element Analysis: Solids and Structures, 157, Springer Science \& Business Media (2008).

27. Liew, K., Xiang, Y., and Kitipornchai, S. "Transverse vibration of thick rectangular plates-I. Comprehensive sets of boundary conditions", Computers \& Structures, 49(1), pp. 1-29 (1993).

28. Hinton, E., Numerical Methods and Software for Dynamic Analysis of Plates and Shells, Swansea: Pineridge Press (1988).

29. Liew, K., Xiang, Y., Kitipornchai, S., et al. "Vibration of thick skew plates based on Mindlin shear deformation plate theory", Journal of Sound and Vibration, 168(1), pp. 39-69 (1993).

30. Kitipornchai, S., Xiang, Y., Liew, K., et al. "A global approach for vibration of thick trapezoidal plates", Computers \& Structures, 53(1), pp. 83-92 (1994).

31. Hosseini-Hashemi, S., Khorshidi, K., and Amabili, M. "Exact solution for linear buckling of rectangular Mindlin plates", Journal of Sound and Vibration, 315(1), pp. 318-342 (2008).

\section{Biographies}

Majid Mohammadi Nia obtained his BSc degree in Civil Engineering from Shahid Bahonar University of Kerman, Kerman, Iran in 2014. He is now an MSc student at Shahid Bahonar University, Iran, where he is studying Structural Engineering. Mr. Mohammadi Nia has published one conference article so far. His research interests include finite element modeling, computational mechanics, and performance based seismic design and evaluation of structures.

Saeed Shojaee has been an Associate Professor of Structural Engineering at Shahid Bahonar University of Kerman, Kerman, Iran since 2008, where he has been teaching advanced engineering mathematics, nonlinear analysis, and control of structures. He earned his BSc degree in Civil Engineering from Shahid Bahonar University, Iran in 2001, his MSc degree in Structural Engineering from Iran University of Science and Technology, Iran in 2003, and his PhD degree in Structural Engineering from Iran University of Science and Technology, Iran in 2008. Dr. Shojaee has published numerous papers in peer-reviewed national and international journals and conference articles. His research interests include optimization techniques, finite and boundary element methods, and computational mechanics.

Saleh Hamzehei-Javaran has been an Assistant Professor of Structural Engineering at Shahid Bahonar University of Kerman, Kerman, Iran since 2014 where he has been teaching theory of elasticity, theory of plates and shells, and continuum mechanics. He earned his BSc degree in Civil Engineering from Shahid Bahonar University, Iran in 2006, his MSc degree in Structural Engineering from Tarbiat Modares University, Iran in 2009, and his PhD degree in Structural Engineering from Tarbiat Modares University, Iran in 2013. Dr. Hamzehei-Javaran has published several papers in peer-reviewed national and international journals and conference articles. His research interests include boundary and finite element methods, computational mechanics, and wave propagation. 